\title{
77TH ANNUAL SASKATCHEWAN CHRISTMAS BIRD COUNT - 2018
}

Alan R. Smith and Randi Edmonds Box 154

Avonlea, SK SOH OCO

alanrandi@sasktel.net

As readers will soon see, the Christmas Bird Count of 2018-19 was one of dullest of the century. Of some interest, however, are two changes to the count. The Gray Jay has officially been renamed the Canada Jay, and the Squaw Rapids Count has been renamed the $E$. $B$. Campbell Dam Count. All Canadians will applaud both changes; our First Nations, in particular, should approve the second.

\section{The Counts}

The number of counts declined by three counts to 86 over last year's 89 . Total effort as measured by hours in the field and at feeders, or kilometres walked and driven was, however, about average for the last 20 years.

\section{The Weather}

Average minimum and maximum temperatures for the count period (with 2017-18 records in brackets) were -10 to $-5 \mathrm{C}(-18$ to $-12 \mathrm{C})$, wind speeds 8 to $19 \mathrm{~km} / \mathrm{h}$ (9 to $18 \mathrm{~km} / \mathrm{h}$ ), and snow depths 6 to $14 \mathrm{~cm}$ (6 to 14 $\mathrm{cm})$. As can be seen, temperatures this past winter were warmer, while wind speeds and snow depths were similar to the previous winter.

\section{The Birds}

The 108,164 birds counted was lower than last winter's 127,798 and the century average of 125,000 . This is partly due to fact that there was no count conducted at Coronach, which usually hosts at least 10,000 waterfowl. The 88 species recorded on count day was the fewest since 1992 when 77 species were tallied. The average number of species per count at 18.2 was, however, on par since 2000.

Regina with 41 species (plus 1 during the count period) won the crown for the most species; the runner up was Gardiner Dam with 40 species.

\section{Population Trends}

Waterfowl were generally found in smaller numbers and fewer localities than normal. Record high counts of Ring-necked Duck and Lesser Scaup were, however, recorded at Gardiner Dam (Table 6).

Most raptors were generally scarce this winter. For example, there were no reports of Northern Harriers and only six Short-eared Owls were seen - all on the Regina Count. Snowy Owls were also harder to find with only 84 in 29 localities compared to 181 in 33 localities the last winter.

The range expansion for two introduced species, Eurasian Collared-Dove and House Finch, seems to have stalled. The only new CBC localities for the dove, Dundurn and White Bear, should be regarded as "infilling" rather than expansion, but the appearance of House Finches at one new locality at Melfort is a $100 \mathrm{~km}$ expansion to the northeast.

Bohemian Waxwings dropped from 12,590 in 33 localities in 2016-17 to 7,469 in 28 localities in 2017-18 to only 3,312 in 22 in 2018-19. This is in spite of the fact that the wild food crop was reported "good" or better in more areas this past winter than in the previous two. Fortunes of another boreal species, the Red-breasted Nuthatch, were much better with 823 birds in 66 localities versus 377 in 44 last winter.

Numbers of the subarctic-nesting Common Redpoll were way down with only 3,412 birds on 55 counts compared to 14,307 on 78 counts in 2017-18. Such "year on year off" fluctuations are, however, the norm for the species. Numbers of most other finches were also down.

\section{Rarities}

The few rarities of note include our sixth Double-crested Cormorant at Gardiner Dam and our fifth Great Blue Heron at Regina. No new species were recorded for the $C B C$; the all-time total remains at 191 species.

Note: for purposes of Saskatchewan Counts as published herein, the count period extends from December 14 to January 5; Audubon counts include only those species recorded three days prior to and after the count day.

\section{Count areas and participants}

(names of compilers are in italics)

1. ARCHERWILL. Sharren Carlson, Shauna Etsell, Gerald Hiron, Susan Hiron, Audrey Hnetka, Perry Hnetka, Elaine Hughes, Dorothy Klettberg, Willie Klettberg, Annette Kozak.

2. AVONLEA. Alan Smith, Blaine Sudom. 
3. BALGONIE. Brian Armstrong, Lionel Bonneville, Joel Cherry, Jared Clarke, Rowan Clarke, Teal Clarke, Ken Feltin, Dale Hjertaas, Phil Holloway, Fran Kerbs, Laurie Koepke, Kristen Martin, Annie McLeod, Brett Quiring, Vera Raschke, Dan Sawatzky, Kathy Schaffer, Scott Schaffer, Hanna Walczykowski, Jacklyn Waronek.

4. BIGGAR. Dale Booth, Ryan Dudragne, Guy Wapple, Rob Wapple.

5. BIRCH HILLS. Margaret Mareschal, Maurice Mareschal.

6. BORDEN-RADISSON. Greg Fenty, Kyron Giroux, Ron Jensen, Jennipher Karst, Scott Olshanoski, Nick Saunders,

7. BROADVIEW. Doug Boivin, Barb Weidl, Don Weidl.

8. CANDLE LAKE. David Britton, Kevin Moore, Vicki St. Germaine, Warren St. Germaine.

9. CATER: Beverly Beland, Orval Beland, Donna Long.

10. CHATSWORTH. Herb Cross, Jean Cross, Charles Dyck, Donna Dyck, Robert Mess, Wionna Mess, George Murray, Laurie Murray, Kerri Rooke, Stewart Rooke, Carol Tangedal, Tony Tangedal.

\section{CHURCHBRIDGE B. Dennis Petracek.}

12. CLARK'S CROSSING. Dale Booth, Dave Cook, Louise Cook, Lorne Duczek, Andrew Elgin, Shelly Fisher, Bob Godwin, Marilyn Haskins, Ron Jensen, Robert Johanson, Gwen Klypak, Heney Klypak, Blair McCann, Brian McGill, Eileen McGill, Diane Murphy, Lynn Oliphant, John Patterson, Sylvia Raginski, Nick Saunders, Jan Shadick, Stan Shadick, Rhonda Shewfelt, Laura Stewart, Marten Stoffel, Guy Wapple, Diane Wells, Michael Williams.
13. CRAVEN. Marla Anderson, Hiroyuki Aoki, Marj Bird, Joel Cherry, Blanche Cooper, Jim Cummings, Stephen Davis, Terry Ford, Jill Forester, Chris Harris, Louise Holloway, Phil Holloway, Andrew Huculak, Bonnie Huculak, Jones Irving, Kerri Irving, Fran Kerbs, Laurie Koepke, Andre Kroeger, Nick Kroeger, Jackie Kuikman, Lauretta Lane, Bob Luterbach, Jeannette Luttmer, Kent Lynn, Barbara Mader, Juan Martinez, Amelia McDonald, Jaret McDonald, Bob McGill, Annie McLeod, Barry Mitschke, Curtis Pollock, Brett Quiring, Vera Raschke, Ed Rodger, Susan Rollins, Jacqueline Roy, Nick Selinger, Jeanette Taylor, Robb Taylor, Lorna Tyler, Chris Whitall, Mary Worel.

14. CROOKED LAKE. Boyd Metzler, Tony Saltasuk.

\section{CROOKED RIVER. Margaret}

Mehler, Morley Mehler.

16. CYPRESS HILLS PROVINCIAL PARK (Centre Block). Dwight Dobson, Nancy Dobson, David Larson, Margarette Larson, Brynne Martin, Mimi Martin, Kevin Moore, Melody Nagel-Hisey.

17. DENHOLM. Beverly Beland, Gerard Beland, Linda Beland, Orval Beland.

18. DORINTOSH. Vicky Pryor, Joe Twidale, Lorraine Twidale, Tim Wahl.

19. DUNDURN. Ron Jensen, Nick Saunders, Phil Taylor, Guy Wapple, Don Weidl.

20. DUVAL. Merv Hey, Lloyd Saul.

\section{E. B. CAMPBELL DAM. Bert} Dalziel, Joan Dalziel, Ryan Dudragne (non-participating compiler), Bill Weighill, Clara Weighill.

22. EASTEND. Loraine Armstrong, Roland Bear, Roxie Binkley, Duane Bristow, Betty Davis, Tom Donald, Cynthia Fehr, Robert Gebhardt, June Higgins, Joan Hodgson, Harvey Johnson, Katherine Myhr, Mary
Thomson, Heidi Topham, Eldon Wig, Jack Wilkinson, Jan Wilkinson.

23. EBENEZER B. Carol Wegner, Harvey Wegner.

\section{ENDEAVOUR. Norman Harris.}

25. ESTEVAN. Barry Dies, Marilyn Dies, Craig Palmer, Larry Preddy, Reid Walton, Rose Walton, Guy Wapple, Sandra Wapple.

26. ESTUARY NORTH. Barry Cocks, Cathy Cocks, Dean Francis.

\section{FENTON. Carman Dodge.}

28. FLORAL. Ryan Dudragne, Guy Wapple.

29. FORT QU'APPELLE. Jean Ashcroft, Peter Ashcroft, Linda Banman, Mark Geremia, Darryl Hamiltin, Alice Isfan, Jack Lowe, Marg Lowe, Jean McKenna, Alan Mlazgar, Wendy Paquin, Keith Stephens, Kim Stephens, Ted Stevenson, Colette Stushnoff.

30. GARDINER DAM. Ryan Dudragne, Andrew Elgin, Greg Fenty, Ron Jensen, Scott Olshanoski, Nick Saunders, Dan Sawatzky, Stan Shadick, Marten Stoffel, Phil Taylor, Guy Wapple.

31. GOOD SPIRIT LAKE. Joyce Anaka, Dorothy Riesz, Ray Riesz.

32. GRASSLANDS NATIONAL PARK. Kevin Moore, Vicki St. Germaine, Warren St. Germaine.

33. GRAYSON. Jack Carrigan, Carina Helm, Charles Helm, Daniel Helm, Linda Helm, Jeanette Zimmer, Karl Zimmer.

34. GRENFELL. Ethel Reiger, Barb Weidl, Don Weidl.

35. HARRIS. Ron Jensen, Guy Wapple, Rob Wapple. 
36. HUDSON BAY. Roger Alain, John Daisley, Caleb Ekerty, Cash Ekert, Meredith Eikert, Alana Gelhorn, Agnes Lewellin, Abby Rawling, Nixon Rawlings, Dennis Reimer, Ruth Reimer, Gloria Stang, John Zolkavich.

37. INDIAN HEAD. Guy Blair, Ron Chambers, Kay Dixon, J. Dixon, Irvin Escott, David Gehl, Roberta Gehl, Ed Haid, Edith Haid, Jim Jinks, Linda Jinks, Dan Loran, H. Madeley, Dora Nichols, Jim Rudack, Ruth Rudack, Lorette Schaeffer, Raymond Schaeffer, Brian Scott, Glenn Scott, Lorne Scott Fred Skinner, C. Skinner.

38. KENASTON. Doug Beckie, $P$. Lawrence Beckie, Theresa Beckie, Patti Hertz.

39. KENOSEE LAKE. Boyd Metzler, John Pollock.

40. KETCHEN NORTH. Dallas Fairburn.

41. KINLOCH. Don Forbes, Carter Haroldson, Robert Haroldson, Darcy Swider, Doreen Wickstrom.

42. KYLE. Greg Nelson, Yvonne Nelson, Marten Stoffel, Dan Zazelenchuk.

43. KUTAWAGEN LAKE. Val Harris, Sheila Lamont

44. LAC LA PLONGE. Erin Conkin, John Conkin, Katherine Conkin.

45. LA RONGE. Alina Mack, Melanie Mack, Serenity Mack, Linda Mikolayenko, Sid Robinson, John Schisler, Jan Shewchuk.

46. LMLNWA. Val Harris, Sheila Lamont

47. LEROY. Brian Galka, Lloyd Saul.
48. LOVE - TORCH RIVER. Bert Dalziel, Joan Dalziel, Sara Dalziel, Scott

Edwards, Andrea Fisher, Harold Fisher, Taren Fisher, Betty Isbister, Henry Isbister, Adrik Kurbis, Coulter Kurbis, Duane Kurbis, Renee Kurbis, Shawn Paschke, Josh Turlte, Leonard Turtle, Shelly Vallier.

49. LUSELAND. Estelle Finley, Graeme Finley, Kim Finley, Liam Finley, Valerie Finley, Brent Honeker.

50. MARTINEAU RIVER. Jan Shadick, Stan Shadick, Vicki St. Germaine, Warren St. Germaine.

51. MEADOW LAKE. Bob Wilson, Ian Wilson.

52. MELFORT. Kirsten Ballantyne, Bert Dalziel, Joan Dalziel, Gordon Dodds, Shirley Dodds, Susan Dodds, Graydon Eskowich, Kim Eskowich, Wendy Eskowich, Scott Green, Cerey Samida, Kirk Samida, Darleen Thompson.

53. MOOSE JAW. Joel Cherry, Chris Harris, Laurie Koepke, Bob Luterbach, Jeff Mander, Annie McLeod, Brett Quiring, Dan Sawatzky.

54. MOOSE MOUNTAIN. Bill Fletcher, Dorothy Fletcher, Doyle Thomas, Val Thomas.

55. MORSE. Elizabeth Enns, Noel Enns, Stella Enns, Mike Francis, Roxanne Hagley, Randy McCulloch, Joel Priebe, Ken Priebe, Myrna Priebe, Lori Wilson.

56. NICOLLE FLATS. Dale Hjertaas, Fran Kerbs, Mary Worel.

57. NIPAWIN. Carol Blenkin, Nancy Budd, Joyce Christiansen, Rick Douslin, Patti Gaertner, Jennette LeCuyer, George Lidster, Jeri McCleary, Peter McCleary, Doug Phillips, Shirley Phillips, Bill Walter, Elaine Walter.

58. NISBET FOREST, NORTHWEST. Sandra Jewell.
59. NISBET FOREST, WEST. Kim Clark, Kiri Clark, Shamara Clark, Suzanne Clark, Brody Holiski.

60. ODESSA. Arden Curts, Denise Curts, Denny Curts.

61. OUTLOOK. Randi Edmonds, Graham Thomson, Alan Smith.

62. PRINCE ALBERT. Jim Bahr, Doug Braaten, Marie Braaten, David Britton, Kim Clark, Shamara Clark, Bert Dalziel, Joan Dalziel, Carman Dodge, Andrea Fisher, Harold Fisher, Taren Fisher, Ham Greenwood, Sandra Jewell, Gwen Klebek, Collin McGuire, Sonnet McGuire, Christine Rye, John Rye, Vicki St. Germaine, Warren St. Germaine, Rhonda Warrener, Don Weidl.

63. PIKE LAKE. Donna Bruce, Dave Cook, Louise Cook, Yvonne Cuttle, Lorne Duczek, Ryan Dudragne, Andrew Elgin, Matthew Frey, Bob Girvan, Bob Godwin, Greg Hutchings, Audrey MacKenzie, Bill MacKenzie, Janine McManus, Joe Monahan, Murray Morgan, John Patterson, Mark Sabourin, Nick Saunders, Nicola Schaefer, Jan Shadick, Stan Shadick, Joe Stookey, Louisa Stuglin, Stephan Stuglin, Phil Taylor, Guy Wapple, Adrian Werner, Michael Williams, Natalia Wycislak.

64. QU'APPELLE. Jean Ashcroft, Peter Ashcroft, Melanie Beattie, Carlie Bennett, Cory Bennett, Stacy Bennett, Christine Blair, Paul Paulhus, Colette Stushnoff, Richard Stushnoff, Frank Veresh.

65. QU'APPELLE VALLEY DAM. Ryan Dudragne, Andrew Elgin, Robert Johanson, Nick Saunders, Jan Shadick, Stan Shadick, Carl Siemens, Hollyce Siemens, Marten Stoffel, Guy Wapple, Michael Williams.

66. RAYMORE. Val Harris, Sheila Lamont. 
67. REGINA. Ingrid Alesich, Marla Anderson, Brian Armstrong, Arleen Arnold, Sameer Bhuyan, Joanne Bonneville, Lionel Bonneville, Stephane Bonneville, Stephane Canevet, Janet Canwood, Lolamae Crawley, Ron Crawley, Al Cullen, Jim Cummings, Elaine Ehmann, Jaquie Fauth, Phyl Fauth, Mary Fields, Ryan Fisher, Terry Ford, Brendan Graham, Chris Harris, JoAnne Harrison, Zach Harrison, Trevor Herriot, Dale Hjertaas, Myrna Hunter, Marie James, Fran Kerbs, Lucille Lipka, Sarah Ludlow, Bob Luterbach, Laurie Koepke, Andre Kroeger, Maureen Lee, Lauren Mang, Kim Mann, Val Mann, Wayne Pepper, Joseph Poissant, Brett Quiring, Chris Ratch, Ed Rodger, Dan Sawatzky, Gary Seib, Nick Selinger, Margaret Skeel, Brian Sterenberg, Frank Switzer, Mary Switzer, Hanna Walczykowski, Lorraine Weidner.

68. ROSCOMMON S.D. Bernice Althouse, Kate Althouse, Ruby Finnie, Brian Irving, Sophie Jankowski, Joan Lillibo, Dianne Sloan, Graham Sloan, Marguerite Sloan.

69. ROULEAU. Stuart Anderson, Patricia Sterzuk.

70. ROUND LAKE (Prince Albert Area) Crystal Frenette.

71. ROUND LAKE (Qu'Appelle Valley). Boyd Metzler, Tony Saltasuk, Mary Ward, Pat Ward.

72. SALTCOATS. Arden Bradford, Olga Brygider, Len Cameron, Muriel Cameron, Walter Farquharson, John Farquharson, Dave Herron, Gloria Herron, Gerri Knudsen, Ron Knudsen, Fern McKay, Earl Upshall, Verda Upshall, Joan Wilson, Rob Wilson.

\section{SASKATCHEWAN LANDING} PROVINCIAL PARK. Marten Stoffel, Dan Zazelenchuk.

74. SASKATCHEWAN RIVER FORKS. Carman Dodge, Don Weidl.
75. SASKATOON. Joanne Adams, Vanessa Amy, Nick Belliveau, Ryan Bradshaw, Anne Brander, Jacquie Christenson, Yvonne Cuttle, Lorne Duczek, Joan Feather, Greg Fenty, Jennifer Fenty, David Forbes, Albert Gerard, Martin Gerard, Denise Giroux, Keith Giroux, Kyron Giroux, Mike Gollop, Jacob Henderson, Lorie Henderson, Terri Jackson, Ron Jensen, Shan Landry, Anna Leighton, Janet Loran, Audrey MacKenzie, Bill MacKenzie, Jane McPhee, Coleen Meldrum, Scott Mitchell, Kelley Moore, Jonathan Moore-Wright, Verity Moore-Wright, Hilda Noton, Jim Paul, John Patterson, Diane Rogers, Marc Sabourin, Monica Sallas, Dick Santo, Trish Santo, Scott Saretsky, Nick Saunders, Andrew Schaaan, Jan Shadick, Stan Shadick, Coy Sharp, Barb Sprigings, Marten Stoffel, Lenore Swystun, Margo Taylor, Phil Taylor, Don Torrie, Guy Wapple, Cathy Watts, Hamish Watts, Trent Watts, George West, Helen Wilkins, Michael Williams, Dan Zazelenchuk, Norman Zlotkin.

\section{SAWYER LAKE. Maureen Blight,} David Weiman.

77. SHAMROCK. Alfred Arnold, Wendy Arnold, Hugh Henry, Iris McNeill, Joel Priebe, Myrna Priebe, Clinton Rud.

78. SHELL LAKE. Ryan Dudragne, Kelly Fairbrother, Carole Martin, Andie Mazur, Kyra Mazur, Beckett Stark, Kay Willson, Phil Willson.

79. SWIFT CURRENT. Jacquie Bolton, Stacy Bolton, Norris Currie, Gordon Dowie, Laurent Dudragne, Mary Ann Dudragne, Arnie Ens, Dave Green, Esther Green, Norma Hain, Hugh Henry, Leonard Howes, Lois Howes, Michelle Hubbard, Ken Knudson, Nicole Kuyek, Connie Lendrum, Dot Letkeman, Robert Moroz, Arden Pierce, Irene Stinson.
80. THICKWOOD HILLS-

SPIRITWOOD. Giles Lalonde, Dawn Mundell, Carole Martins, Kay Willson, Philip Willson.

81. TURTLE LAKE. David Forbes, Reg Forbes, Fred Hegelton, Jackie Hegelton, Brent Keen, Cheryl Robbins.

82. TURTLEFORD. Hank deGraaf, Miles Johnson, Brent Keen, Louise Lundberg, Ron Perkins, Richard Roney, Margaret Uhlig.

83. WEYBURN. Bob Cameron, Lucille Cameron, Darcy Dyck, Al Fahlman, Jo-Ann Fahlman, Glen Fleming, Millie Fleming, Dale Huff, Sandy Huff, Alma McCormick, Morley McCormick, Charlotte Payak, Cole Payak, Don Payak, Janice Phillips, Garnet Schultz, Lina Schultz, Doyle Thomas, Tanis Thomas, Val Thomas, Kim Thorson, Myrt Thorson, Dorothy Whitell, John Whitell.

84. WHITE BEAR. Dan Zazelenchuk.

85. WHITEWOOD. Ken Aldous, Carole Armstrong, Cindy Ashfield, Joe Ashfield, Paul Ashfield, Kerri Bachtold, Joyce Kydd, Sarah Mambourg, Boyd Metzler, Harry Mitchell, Marilyn Mitchell, Donna Mohr, Margaret Niemenen, Paul Niemenen, Tony Saltasuk, Doug Shepherd, Dawn Vennard, Diane Veresh, Pat Ward.

86. WINGARD. Rebecca Beam. 
TABLE 1. Weather and Snow Cover.

\begin{tabular}{|c|c|c|c|c|c|c|c|c|c|}
\hline LOCALITY & DATE & $\begin{array}{c}\text { MIN } \\
\text { TEMP } \\
\left({ }^{\circ} \mathrm{C}\right)\end{array}$ & 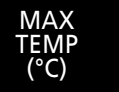 & $\begin{array}{c}\text { MIN } \\
\text { WIND } \\
(\mathrm{KM} / \mathrm{HR})\end{array}$ & $\begin{array}{c}\text { MAX } \\
\text { WIND } \\
(\mathrm{KM} / \mathrm{HR})\end{array}$ & $\begin{array}{l}\text { MIN } \\
\text { SMOW } \\
\text { (CM) }\end{array}$ & $\begin{array}{c}\text { MAX } \\
\text { SNOW } \\
\text { (CM) }\end{array}$ & SKY A.M. & SKY P.M. \\
\hline Archerwill & 28 Dec 2018 & -20 & -18 & 10 & 17 & 8 & 8 & partly cloudy & partly cloudy \\
\hline Avonlea & 2 Jan 2019 & -8 & -6 & 0 & 20 & 0 & 10 & partly cloudy & cloudy \\
\hline Balgonie & 5 Jan 2019 & -8 & -4 & 20 & 40 & 5 & 5 & overcast & partly cloudy \\
\hline Biggar & 20 Dec 2018 & -8 & -4 & 5 & 13 & 0 & 10 & overcast & partly cloudy \\
\hline Birch Hills & 4 Jan 2019 & -5 & -2 & 2 & 5 & 4 & 30 & partly cloudy & partly cloudy \\
\hline Borden-Radisson & 21 Dec 2018 & -3 & 3 & 10 & 52 & & & & \\
\hline Broadview & 28 Dec 2018 & -23 & -17 & 5 & 10 & 4 & 6 & clear & mostly clear \\
\hline Candle Lake & 5 Jan 2019 & -14 & -13 & 1 & 5 & 15 & 30 & overcast & light snow \\
\hline Cater & 3 Jan 2019 & -4 & 0 & 0 & 8 & 10 & 20 & partly cloudy & clear \\
\hline Chatsworth S.D. & 4 Jan 2019 & -8 & 2 & 1 & 1 & 2 & 16 & partly cloudy & partly cloudy \\
\hline Churchbridge B & 5 Jan 2019 & & & & & & & overcast & overcast \\
\hline Clark's Crossing & 15 Dec 2018 & 1 & 4 & 22 & 40 & 0 & 15 & partly cloudy & mostly clear \\
\hline Craven & 15 Dec 2018 & -2 & 3 & 19 & 33 & 0 & 6 & overcast & mostly clear \\
\hline Crooked Lake & 2 Jan 2019 & -3 & -1 & 10 & 20 & 2 & 15 & cloudy & partly cloudy \\
\hline Crooked River & 27 Dec 2018 & -20 & & 2 & 11 & & & mostly clear & mostly clear \\
\hline Cypress Hills P.P. & 28 Dec 2018 & -8 & -5 & 2 & 5 & 5 & 10 & mostly clear & mostly clear \\
\hline Denholm & 25 Dec 2018 & -16 & -12 & 0 & 6 & 4 & 10 & partly cloudy & partly cloudy \\
\hline Dorintosh & 22 Dec 2018 & -9 & -3 & 2 & 5 & 20 & 30 & mod. snow & light snow \\
\hline Dundurn & 22 Dec 2018 & -9 & -5 & 28 & 38 & 0 & 10 & overcast & light snow \\
\hline Duval & 26 Dec 2018 & -14 & -7 & 0 & 10 & 0 & 22 & clear & partly cloudy \\
\hline E.B.Campbell Dam & 3 Jan 2019 & 0 & 3 & 10 & 25 & 25 & 10 & cloudy & partly cloudy \\
\hline Eastend & 3 Jan 2019 & 1 & 1 & 15 & 40 & 0 & 10 & partly cloudy & partly cloudy \\
\hline Ebenezer B & 29 Dec 2018 & -10 & -5 & 10 & 15 & 6 & 12 & cloudy & cloudy \\
\hline Endeavour & 28 Dec 2018 & -14 & -8 & 2 & 5 & & & cloudy & cloudy \\
\hline Estevan & 30 Dec 2018 & -13 & -10 & 22 & 30 & 5 & 15 & light snow & light snow \\
\hline Estuary North & 27 Dec 2018 & -12 & -13 & 10 & 30 & 0 & 0 & overcast & light snow \\
\hline Fenton & 4 Jan 2019 & -4 & -10 & & & & & mostly clear & mostly clear \\
\hline Floral & 14 Dec 2018 & -6 & 4 & 16 & 25 & 0 & 5 & mostly clear & partly cloudy \\
\hline Fort Qu'Appelle & 14 Dec 2018 & 0 & 2 & 2 & 2 & 0 & 2 & clear & clear \\
\hline Gardiner Dam & 17 Dec 2018 & -6 & -4 & 10 & 18 & 0 & 10 & partly cloudy & partly cloudy \\
\hline Good Spirit Lake & 4 Jan 2019 & 1 & 3 & 15 & 20 & 5 & 25 & partly cloudy & mostly clear \\
\hline Grasslands N.P. & 19 Dec 2018 & -2 & 0 & 2 & 5 & 1 & 5 & mostly clear & mostly clear \\
\hline Grayson & 27 Dec 2018 & -12 & -10 & 0 & 10 & 0 & 10 & light snow & light snow \\
\hline Grenfell & 5 Jan 2019 & -5 & -4 & 0 & 5 & 2 & 5 & light fog & mostly clear \\
\hline Harris & 19 Dec 2018 & & & & & & & mostly clear & mostly clear \\
\hline Hudson Bay & 29 Dec 2018 & -18 & -15 & 15 & 20 & 25 & 30 & cloudy & \\
\hline Indian Head & 27 Dec 2018 & -24 & -15 & 5 & 10 & 8 & 8 & clear & partly cloudy \\
\hline Kenaston & 15 Dec 2018 & 1 & 3 & 5 & 20 & 1 & 4 & partly cloudy & clear \\
\hline Kenosee Lake & 27 Dec 2018 & -13 & -11 & 6 & 25 & 5 & 15 & light snow & overcast \\
\hline Ketchen North & 26 Dec 2018 & -14 & -12 & 0 & 10 & 12 & 16 & overcast & cloudy \\
\hline Kinloch & 27 Dec 2018 & -18 & -10 & 15 & 20 & 23 & 26 & partly cloudy & overcast \\
\hline Kutawagen Lake & 4 Jan 2019 & -6 & -3 & 10 & 30 & 0 & 10 & partly cloudy & partly cloudy \\
\hline Kyle & 29 Dec 2018 & -11 & -4 & 0 & 2 & 0 & 2 & clear & overcast \\
\hline Lac La Plonge & 28 Dec 2018 & -15 & -14 & 0 & 5 & 25 & 50 & overcast & mod. snow \\
\hline La Ronge & 2 Jan 2019 & -14 & -11 & 30 & 39 & 22 & 25 & cloudy & partly cloudy \\
\hline
\end{tabular}




\begin{tabular}{|c|c|c|c|c|c|c|c|c|c|}
\hline LOCALITY & DATE & $\begin{array}{c}\text { MIN } \\
\text { TEMP } \\
\left({ }^{\circ} \mathrm{C}\right)\end{array}$ & $\begin{array}{c}\text { MAX } \\
\text { TEMP } \\
\left({ }^{\circ} \mathrm{C}\right)\end{array}$ & $\begin{array}{c}\text { MIN } \\
\text { WIND } \\
\text { (KM/HR) }\end{array}$ & 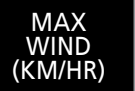 & $\begin{array}{c}\text { MIN } \\
\text { SMOW } \\
\text { (CM) }\end{array}$ & $\begin{array}{l}\text { MAX } \\
\text { SNOW } \\
\text { (CM) }\end{array}$ & SKY A.M. & SKY P.M. \\
\hline LMLNWA & 5 Jan 2019 & -10 & -3 & 8 & 10 & 0 & 10 & overcast & overcast \\
\hline Leroy & 5 Jan 2019 & -10 & -8 & 0 & 5 & 0 & 17 & light fog & overcast \\
\hline Love-Torch River & 26 Dec 2018 & -25 & -12 & 0 & 5 & & & overcast & overcast \\
\hline Luseland & 22 Dec 2018 & -7 & -4 & 20 & 29 & 2 & 6 & cloudy & cloudy \\
\hline Martineau River & 21 Dec 2018 & -3 & -2 & 21 & 31 & 4 & 6 & light snow & mod. snow \\
\hline Meadow Lake & 26 Dec 2018 & -12 & -10 & 20 & 40 & 10 & 20 & cloudy & light snow \\
\hline Melfort & 22 Dec 2018 & -30 & -26 & 13 & 26 & & & clear & clear \\
\hline Moose Jaw & 22 Dec 2018 & -5 & -5 & 15 & 44 & 0 & 5 & overcast & overcast \\
\hline Moose Mountain & 29 Dec 2018 & -14 & -7 & 2 & 4 & 4 & 10 & partly cloudy & partly cloudy \\
\hline Morse & 16 Dec 2018 & -10 & -3 & 10 & 20 & 0 & 30 & overcast & partly cloudy \\
\hline Nicolle Flats & 19 Dec 2018 & -1 & 3 & 5 & 25 & 0 & 6 & partly cloudy & mostly clear \\
\hline Nipawin & 26 Dec 2018 & -16 & -11 & 0 & 5 & 15 & 15 & cloudy & cloudy \\
\hline Nisbet Forest NW & 26 Dec 2018 & -16 & -7 & 2 & 6 & 9 & & light fog & light snow \\
\hline Nisbet Forest West & 29 Dec 2018 & -16 & -16 & 10 & 20 & 20 & 20 & overcast & overcast \\
\hline Odessa & 29 Dec 2018 & -10 & -4 & 10 & 15 & 4 & 10 & overcast & partly cloudy \\
\hline Outlook & $26 \operatorname{Dec} 2018$ & -10 & -8 & 0 & 10 & 0 & 5 & partly cloudy & partly cloudy \\
\hline Pike Lake & 5 Jan 2019 & -7 & 0 & 10 & 24 & 2 & 15 & partly cloudy & cloudy \\
\hline Prince Albert & 16 Dec 2018 & -15 & -15 & 5 & 6 & 4 & 10 & overcast & overcast \\
\hline Qu'Appelle & 28 Dec 2018 & -24 & -12 & 10 & 25 & 5 & 10 & clear & light snow \\
\hline Qu'Appelle Dam & 16 Dec 2018 & -11 & -6 & 0 & 15 & 0 & 10 & cloudy & partly cloudy \\
\hline Raymore & 25 Dec 2018 & -13 & -10 & 6 & 11 & 0 & 15 & light snow & light snow \\
\hline Regina & 29 Dec 2018 & -11 & -5 & 2 & 15 & 5 & 10 & mostly clear & mostly clear \\
\hline Roscommon S.D. & 2 Jan 2019 & -8 & -1 & 10 & 15 & 26 & 31 & overcast & overcast \\
\hline Rouleau & 17 Dec 2018 & -5 & 2 & 20 & 29 & 1 & 2 & partly cloudy & cloudy \\
\hline Round Lake (P.A.) & 5 Jan 2019 & -8 & & 10 & 15 & 10 & 20 & overcast & light snow \\
\hline Round Lake (Q.V.) & 17 Dec 2018 & -10 & -5 & 12 & 25 & 0 & 12 & overcast & overcast \\
\hline Saltcoats & 18 Dec 2018 & -7 & -1 & 12 & 17 & 3 & 5 & clear & clear \\
\hline Sask. Landing P.P. & 20 Dec 2018 & -7 & 1 & 0 & 20 & 0 & 2 & clear & partly cloudy \\
\hline Sask. River Forks & 15 Dec 2018 & 3 & 2 & 5 & 10 & 5 & 10 & mostly clear & clear \\
\hline Saskatoon & 26 Dec 2018 & -12 & -9 & 2 & 7 & 2 & 15 & partly cloudy & partly cloudy \\
\hline Sawyer Lake & 24 Dec 2018 & -14 & -12 & 10 & 20 & 10 & 15 & light snow & \\
\hline Shamrock & 19 Dec 2018 & -2 & 2 & 30 & 45 & 2 & 20 & cloudy & mostly clear \\
\hline Shell Lake & 18 Dec 2018 & -11 & -1 & 0 & 17 & 2 & 15 & partly cloudy & partly cloudy \\
\hline Swift Current & 15 Dec 2018 & 3 & 4 & 35 & 65 & 5 & 20 & partly cloudy & mostly clear \\
\hline $\begin{array}{l}\text { Thickwood- } \\
\text { Spiritwood }\end{array}$ & 21 Dec 2018 & -2 & 2 & 10 & 40 & 0 & 4 & cloudy & overcast \\
\hline Turtle Lake & 27 Dec 2018 & -23 & -20 & 5 & 12 & 10 & 40 & clear & overcast \\
\hline Turtleford & 28 Dec 2018 & -12 & -8 & 0 & 5 & 5 & 10 & cloudy & cloudy \\
\hline Weyburn & 15 Dec 2018 & 0 & 3 & 5 & 8 & 4 & 6 & clear & partly cloudy \\
\hline White Bear & 2 Jan 2019 & -1 & 4 & 15 & 25 & 8 & 2 & overcast & overcast \\
\hline Whitewood & $29 \operatorname{Dec} 2018$ & -15 & -6 & 5 & 20 & 5 & 20 & overcast & overcast \\
\hline Wingard & 2 Jan 2019 & & & & & & & & \\
\hline White Bear & $21 \mathrm{Dec} 2017$ & -12 & -7 & 12 & 19 & 0 & 1 & cloudy & overcast \\
\hline Whitewood & 27 Dec 2017 & -31 & -20 & 20 & 39 & 4 & 10 & overcast & partly cloudy \\
\hline Wingard & 3 Jan 2018 & & & & & & & & \\
\hline
\end{tabular}


TABLE 2. Effort and Habitat Coverage (\%). Wild Fruit: $p=$ poor, $f=f a i r, g=g o o d, e=e x c e l l e n t$.

\begin{tabular}{|c|c|c|c|c|c|c|c|c|c|c|c|c|c|c|c|c|c|c|c|c|}
\hline LOCALITY & 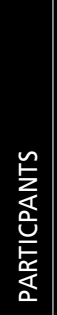 & 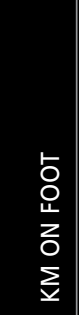 & 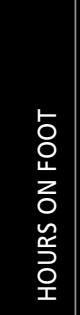 & 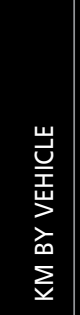 & 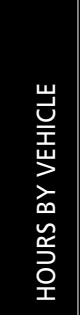 & 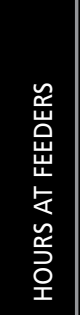 & 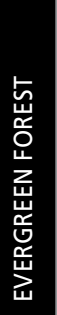 & 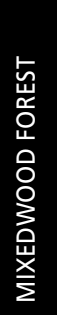 & 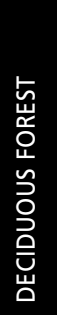 & 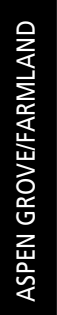 & 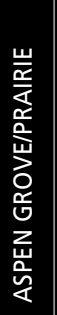 & 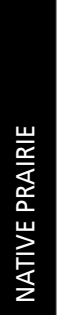 & 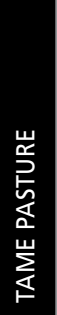 & 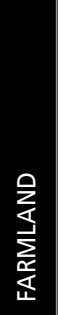 & 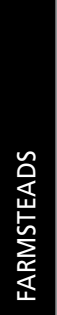 & $\begin{array}{l}z \\
\text { zo } \\
\text { ơ } \\
\text { s. }\end{array}$ & 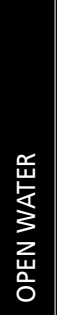 & $\begin{array}{l}\frac{z}{\alpha} \\
\frac{\alpha}{a} \\
\frac{a}{\alpha}\end{array}$ & 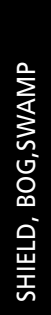 & 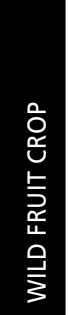 \\
\hline Archerwill & 10 & 0.5 & 0.5 & 0 & 0.0 & 8.0 & & 25 & & & & & & 50 & 25 & & & & & o \\
\hline Avonlea & 2 & 0.0 & 0.0 & 141 & 6.0 & 1.3 & & & 5 & & & 5 & 5 & 25 & 5 & 50 & & 5 & & $f$ \\
\hline Balgonie & 19 & 36.0 & 12.5 & 492 & 19.5 & 0.0 & & & & 20 & & & & 45 & 10 & 25 & & & & $p$ \\
\hline Biggar & 9 & 7.0 & 5.8 & 264 & 11.0 & 7.0 & & & & 15 & & & & 40 & 10 & 35 & & & & $f$ \\
\hline Birch Hills & 2 & 0.5 & 0.5 & 51 & 5.5 & 1.0 & & & & 20 & & & 10 & 45 & 5 & & & 20 & & $f$ \\
\hline Borden-Radisson & 6 & 14.0 & 7.0 & 236 & 6.5 & 0.0 & & & & & & & & & & & & & & \\
\hline Broadview & 3 & 1.5 & 1.0 & 136 & 5.5 & 2.0 & & & 20 & 25 & & 5 & 10 & 25 & 5 & 10 & & & & $\mathrm{p}$ \\
\hline Candle Lake & 4 & 6.0 & 1.5 & 124 & 8.2 & 0.0 & & 70 & & & & & & & 25 & & & & 5 & $\mathrm{f}$ \\
\hline Cater & 3 & 0.0 & 0.0 & 224 & 8.0 & 1.0 & 5 & 10 & 5 & 15 & & & 5 & 50 & 5 & 5 & & & & $\mathrm{~g}$ \\
\hline Chatsworth S.D. & 12 & 2.0 & 8.0 & 98 & 6.0 & 5.0 & & & & 80 & & 10 & 5 & & 5 & & & & & $\mathrm{p}$ \\
\hline Churchbridge B & 1 & & & & & 8.0 & & & & & & & & & & 100 & & & & $\mathrm{p}$ \\
\hline Clark's Crossing & 29 & 15.0 & 13.3 & 600 & 29.4 & 5.5 & & & & 30 & 5 & & & 20 & 15 & 20 & & 10 & & $\mathrm{f}$ \\
\hline Craven & 45 & 29.8 & 15.2 & 609 & 23.9 & 13.5 & & & & 25 & 15 & 5 & 4 & 20 & 10 & 20 & & & & $f$ \\
\hline Crooked Lake & 2 & 0.0 & 0.0 & 131 & 5.0 & 0.0 & & & & 10 & 15 & & & 40 & 5 & 20 & 10 & & & $f$ \\
\hline Crooked River & 2 & 0.0 & 0.0 & 15 & 1.0 & 0.0 & & 40 & & & & & & 40 & 20 & & & & & $f$ \\
\hline Cypress Hills P.P. & 8 & 8.0 & 5.0 & 15 & 1.0 & 0.0 & 40 & 40 & 8 & & & 10 & & & 2 & & & & & $\mathrm{~g}$ \\
\hline Denholm & 4 & 0.0 & 0.0 & 224 & 6.5 & 1.0 & & & & 10 & 5 & & 5 & 70 & 5 & 5 & & & & e \\
\hline Dorintosh & 4 & 8.0 & 3.0 & 32 & 1.0 & 0.7 & & 60 & & & & & & & 40 & & & & & $\mathrm{f}$ \\
\hline Dundurn & 4 & 4.0 & 2.0 & 136 & 4.3 & 0.0 & & & & 30 & & & & 60 & & 10 & & & & $\mathrm{~g}$ \\
\hline Duval & 2 & 2.0 & 1.0 & 107 & 6.8 & 0.3 & & & & 20 & 5 & & & 35 & 10 & 30 & & & & $\mathrm{~g}$ \\
\hline E.B.Campbell Dam & 4 & 2.0 & 1.0 & 92 & 4.5 & 2.0 & & 70 & & & & & & & 10 & & 20 & & & $\mathrm{p}$ \\
\hline Eastend & 17 & 20.0 & 10.0 & 200 & 30.0 & 0.0 & & 5 & & 20 & 50 & & 5 & 5 & & 15 & $<1$ & & & $\mathrm{~g}$ \\
\hline Ebenezer B & 2 & 0.0 & 0.0 & 28 & 4.0 & 3.0 & & 5 & & & & 50 & 30 & 10 & & 5 & & & & $\mathrm{p}$ \\
\hline Endeavour & 1 & 0.0 & 0.0 & 15 & 2.0 & 4.0 & & & & 40 & & & & & 60 & & & & & $\mathrm{f}$ \\
\hline Estevan & 8 & 2.5 & 2.3 & 196 & 6.8 & 5.0 & & & & & & & & 40 & 10 & 20 & 15 & 15 & & $f$ \\
\hline Estuary North & 3 & & 3.0 & & 15.0 & 3.0 & & & 30 & & & 30 & & 30 & 10 & & & & & e \\
\hline Fenton & 1 & 0.0 & 0.0 & 80 & 3.3 & 0.0 & & & 10 & & & 20 & 20 & 40 & 10 & & & & & $\mathrm{~g}$ \\
\hline Floral & 2 & 4.0 & 3.8 & 203 & 5.3 & 0.0 & & & & 15 & & & & 50 & 10 & 25 & & & & $f$ \\
\hline Fort Qu'Appelle & 15 & 0.0 & 0.0 & 287 & 16.0 & 0.0 & & & & 25 & 20 & & 10 & 20 & 5 & 20 & & & & $\mathrm{p}$ \\
\hline Gardiner Dam & 11 & 12.0 & 10.8 & 381 & 17.0 & 0.0 & & & & 5 & & & & 50 & 10 & 10 & 25 & & & $\mathrm{f}$ \\
\hline Good Spirit Lake & 3 & 0.0 & 0.0 & 124 & 6.0 & 0.0 & & & & 20 & & & 20 & 30 & 5 & 25 & & & & $\mathrm{p}$ \\
\hline Grasslands N.P. & 3 & 2.5 & 1.0 & 281 & 21.0 & 0.0 & & & & & & 85 & 10 & & & 5 & & & & $\mathrm{f}$ \\
\hline Grayson & 7 & 14.0 & 3.0 & 99 & 4.0 & 4.0 & & & & 35 & 5 & & & 25 & 10 & 15 & 5 & 5 & & $f$ \\
\hline Grenfell & 3 & 0.0 & 0.0 & 117 & 4.5 & 2.5 & & & & 15 & 15 & & 5 & 15 & 25 & 10 & 15 & & & $\mathrm{p}$ \\
\hline Harris & 4 & 4.0 & 4.0 & 271 & 10.3 & 0.0 & & & & 5 & & & & 70 & 5 & 20 & & & & $f$ \\
\hline Hudson Bay & 14 & 2.0 & 0.5 & 20 & 1.5 & 10.0 & & 40 & & 40 & & & & & & 20 & & & & $\mathrm{~g}$ \\
\hline Indian Head & 22 & & 2.0 & 418 & 10.0 & 17.0 & 5 & & & 10 & & & & 75 & 5 & 5 & & & & $\mathrm{p}$ \\
\hline Kenaston & 3 & 0.0 & 0.0 & 216 & 6.0 & 0.0 & & & & & & & & 90 & 5 & 5 & & & & \\
\hline Kenosee Lake & 2 & & 0.5 & 143 & 4.0 & 0.5 & & & 20 & 10 & 10 & 5 & 5 & & & 50 & & & & $\mathrm{f}$ \\
\hline Ketchen North & 1 & 1.0 & 1.0 & 50 & 2.5 & 6.0 & & & & 30 & & & 20 & 20 & 20 & 10 & & & & \\
\hline Kinloch & 5 & 2.0 & 1.0 & 128 & 7.0 & 9.0 & 5 & 30 & 20 & 30 & & & & & 15 & & & & & $\mathrm{p}$ \\
\hline Kutawagen Lake & 2 & 0.5 & 0.3 & 147 & 5.7 & 0.7 & & & & 10 & 5 & 5 & & 60 & 10 & 10 & & & & $\mathrm{~g}$ \\
\hline Kyle & 4 & 5.0 & 3.0 & 294 & 12.0 & 0.0 & & & & & & 15 & 5 & 30 & 20 & 15 & & 15 & & $\mathrm{f}$ \\
\hline Lac La Plonge & 3 & 2.0 & 1.0 & 40 & 4.0 & 0.5 & 25 & 35 & 25 & & & & & & & 15 & & & & $\mathrm{~g}$ \\
\hline La Ronge & 7 & 3.0 & 4.5 & 68 & 6.0 & 2.5 & & 20 & & & & & & & & 70 & & 10 & & $\mathrm{~g}$ \\
\hline
\end{tabular}




\begin{tabular}{|c|c|c|c|c|c|c|c|c|c|c|c|c|c|c|c|c|c|c|c|c|}
\hline LOCALITY & 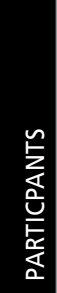 & 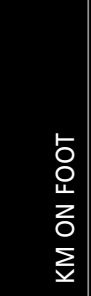 & $\begin{array}{l}5 \\
\text { 인 } \\
\text { z } \\
\text { o } \\
\text { 똑 } \\
\text { 우 }\end{array}$ & 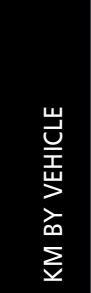 & 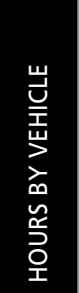 & 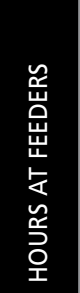 & 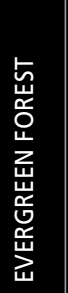 & 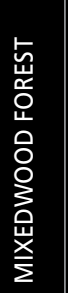 & 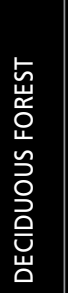 & 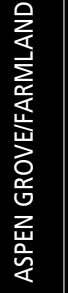 & 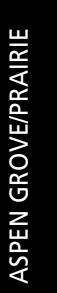 & 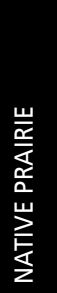 & 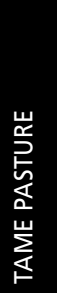 & 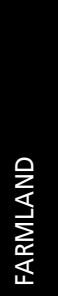 & 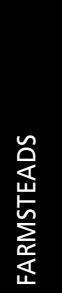 & 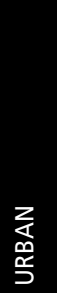 & $\begin{array}{l}\text { 妥 } \\
3 \\
z \\
\text { 岕 } \\
0\end{array}$ & $\begin{array}{l}\frac{z}{a} \\
\frac{\alpha}{\alpha} \\
\frac{\bar{c}}{\bar{x}}\end{array}$ & 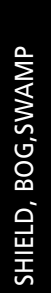 & 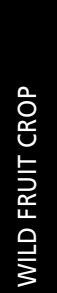 \\
\hline LMLNWA & 2 & 0.5 & 0.5 & 175.2 & 7.8 & 1.0 & & & & 10 & & 10 & & 40 & 10 & 25 & 5 & & & $e$ \\
\hline Leroy & 2 & 2.0 & 1.0 & 149 & 6.0 & 0.5 & & & & 15 & & & & 35 & 15 & 35 & & & & $f$ \\
\hline Love-Torch River & 20 & 4.8 & 4.3 & 411 & 22.0 & 14.0 & 20 & 10 & & 50 & & & & & 10 & 10 & & & & $\mathrm{p}$ \\
\hline Luseland & 6 & 13.0 & 6.0 & 337 & 7.0 & 3.0 & & & & 50 & & 5 & 5 & 25 & 5 & 10 & & & & $g$ \\
\hline Martineau River & 4 & 0.0 & 0.0 & 45 & 6.8 & 0.0 & & 95 & & 50 & & & & & & & & 5 & & $g$ \\
\hline Meadow Lake & 2 & 5.0 & 1.0 & 125 & 3.3 & 0.0 & & 50 & & & & & & 40 & & 10 & & & & $\mathrm{~g}$ \\
\hline Melfort & 13 & 0.0 & 0.0 & 194 & 7.8 & 16.8 & & & & 5 & & & & 45 & & 50 & & & & $f$ \\
\hline Moose Jaw & 8 & 23.3 & 8.6 & 337 & 13.0 & 0.0 & & 10 & & & & & 5 & 45 & & 30 & & 10 & & $\mathrm{~g}$ \\
\hline Moose Mountain & 4 & 0.0 & 0.0 & 110 & 5.0 & 3.0 & & 55 & & & & 5 & & 25 & 5 & 10 & & & & g \\
\hline Morse & 10 & 3.7 & 1.5 & 494 & 21.5 & 0.5 & & & & & & & 5 & 90 & 5 & & & & & g \\
\hline Nicolle Flats & 3 & 13.6 & 4.0 & 210 & 11.3 & 0.0 & & & 30 & & & 10 & 20 & 30 & & 5 & & 5 & & g \\
\hline Nipawin & 14 & 1.0 & 0.8 & 163 & 5.8 & 8.0 & 15 & 5 & & 15 & & & & 25 & 10 & 30 & & & & $p$ \\
\hline Nisbet Forest NW & 1 & 1.0 & 0.8 & 0 & 0.0 & 3.2 & & 50 & & & & & & & 50 & & & & & \\
\hline Nisbet Forest West & 5 & 0.5 & 1.0 & 100 & 7.0 & 6.0 & 20 & & 20 & 10 & & & & 40 & & 10 & & & & $f$ \\
\hline Odessa & 3 & & 2.0 & & 8.0 & 4.0 & & & & 20 & & 20 & 20 & 20 & 20 & & & & & $f$ \\
\hline Outlook & 3 & 0.0 & 0.0 & 170 & 5.5 & 1.0 & & & & & & 5 & & 45 & 5 & 40 & 5 & & & e \\
\hline Pike Lake & 30 & 23.0 & 23.7 & 393 & 21.2 & 3.0 & & & 10 & 45 & 5 & & & 20 & 15 & 5 & & & & $\mathrm{p}$ \\
\hline Prince Albert & 23 & 24.5 & 12.3 & 420 & 26.1 & 4.0 & & & & & & & & & & & & & & $g$ \\
\hline Qu'Appelle & 11 & 2.5 & 0.8 & 316 & 11.2 & 17.0 & & & & 60 & 5 & & 5 & 20 & 5 & 5 & & & & $\mathrm{p}$ \\
\hline Qu'Appelle Dam & 11 & 8.0 & 8.5 & 548 & 20.0 & 0.0 & & & & 30 & 10 & & & 20 & 10 & 15 & 5 & 10 & & $f$ \\
\hline Raymore & 2 & 0.5 & 0.5 & 131 & 7.8 & 1.0 & & & & 25 & & & 5 & 35 & 10 & 25 & & & & g \\
\hline Regina & 52 & 109.5 & 42.3 & 445 & 20.5 & 11.5 & & & & 10 & & & & 25 & 5 & 60 & & & & $\mathrm{p}$ \\
\hline Roscommon S.D. & 9 & 0.0 & 0.0 & 84 & 3.0 & 24.0 & & & & 20 & & & & 60 & 20 & & & & & $\mathrm{p}$ \\
\hline Rouleau & 2 & 0.0 & 0.0 & 231 & 6.4 & 1.0 & & & & & 5 & & & 80 & 5 & 10 & & & & $p$ \\
\hline Round Lake (P.A.) & 1 & 1.0 & 2.0 & 0 & 0.0 & 5.5 & & 25 & & & & & 25 & 40 & & & & 10 & & $\mathrm{p}$ \\
\hline Round Lake (Q.V.) & 4 & 0.0 & 0.0 & 253 & 5.5 & 1.0 & & & & 15 & & & & 40 & 10 & 25 & 10 & & & \\
\hline Saltcoats & 15 & 0.0 & 0.0 & 60 & 8.0 & 10.0 & & & & 55 & & 10 & & & 20 & 15 & & & & $\mathrm{p}$ \\
\hline Sask. Landing P.P. & 2 & 5.0 & 2.0 & 299 & 7.0 & 0.0 & & & & 5 & & & 10 & 35 & 20 & 5 & & 25 & & $f$ \\
\hline Sask. River Forks & 2 & 2.0 & 1.0 & 83 & 5.3 & 0.0 & 15 & 20 & 20 & 15 & & & 5 & 15 & 5 & & 5 & & & $\mathrm{p}$ \\
\hline Saskatoon & 61 & 89.0 & 46.3 & 772 & 47.2 & 15.9 & & & & 10 & & & & 5 & & 70 & 5 & 10 & & $\mathrm{p}$ \\
\hline Sawyer Lake & 2 & 6.0 & 4.0 & 21 & 4.0 & 0.0 & & 50 & & 10 & & & 20 & & 10 & & & 10 & & $f$ \\
\hline Shamrock & 7 & 4.0 & 3.0 & 391 & 13.5 & 1.0 & & & & & & 5 & 15 & 60 & 15 & 5 & & & & \\
\hline Shell Lake & 8 & 2.5 & 2.5 & 225 & 8 & 0 & 5 & $<1$ & 25 & 5 & 5 & 5 & 30 & 15 & $<1$ & 5 & & 5 & & $f$ \\
\hline Swift Current & 22 & 29.0 & 13.5 & 469 & 27.0 & 6.8 & & & & & & & & 20 & 5 & 70 & & 5 & & $f$ \\
\hline $\begin{array}{l}\text { Thickwood- } \\
\text { Spiritwood }\end{array}$ & 5 & 3.0 & 1.5 & 147 & 6.0 & 2.5 & 5 & 10 & & 25 & & & 10 & 30 & 5 & 10 & & 5 & & $f$ \\
\hline Turtle Lake & 6 & 4.0 & 2.5 & 45 & 2.0 & 0.0 & 20 & 40 & & & & & & 40 & & & & & & $f$ \\
\hline Turtleford & 7 & 12.0 & 3.0 & 410 & 7.0 & 7.0 & & & & 45 & & & & 50 & & 5 & & & & $f$ \\
\hline Weyburn & 24 & 0.0 & 0.0 & 320 & 15.0 & 9.0 & & & 5 & & 5 & 5 & 5 & 60 & 10 & 10 & & & & $f$ \\
\hline White Bear & 1 & 2.0 & 1.0 & 277 & 7.0 & 0.0 & & & & & & 5 & 5 & 65 & 20 & & & 5 & & $f$ \\
\hline Whitewood & 22 & 2.5 & 3.0 & 494 & 14.0 & 37.0 & & & & 5 & & & & 15 & 5 & 75 & & & & $f$ \\
\hline Wingard & 1 & & & & & & & & & & & & & & & & & & & \\
\hline
\end{tabular}


TABLE 3. Species found on 6 or more counts; ( ) = seen during count period (CP); CP totals include CP birds listed in Table 4.

\begin{tabular}{|c|c|c|c|c|c|c|c|c|c|c|c|c|c|c|c|c|c|c|c|c|c|c|c|c|}
\hline SPECIES & 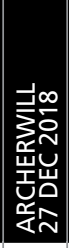 & 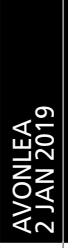 & 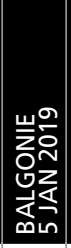 & 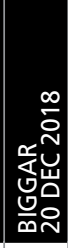 & 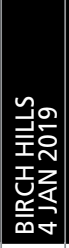 & 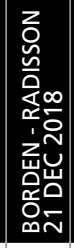 & 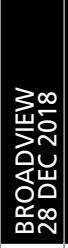 & 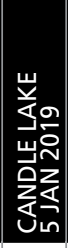 & 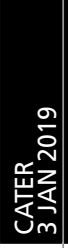 & 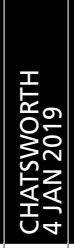 & 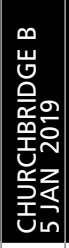 & 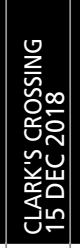 & 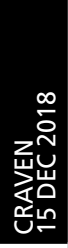 & 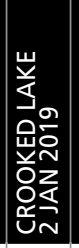 & 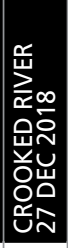 & 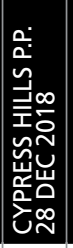 & 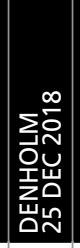 & 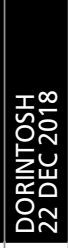 & 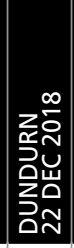 & 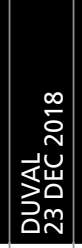 & 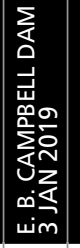 & 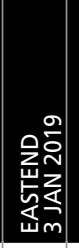 & 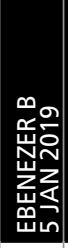 & 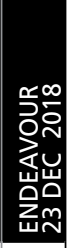 \\
\hline Canada Goose & & & & & & & & & & & & 483 & & 1 & & & & & 54 & & 8 & & & 1 \\
\hline Mallard & & & & & & & & & & & & 2 & & 4 & & & & & & & & & & \\
\hline Common Goldeneye & & & & & & & & & & & & 242 & & 1 & & & & & & & 313 & & & \\
\hline Gray Partridge & & 7 & 40 & & & 28 & 6 & & 6 & & & 94 & 12 & & & & 7 & & 6 & (8) & & & & 13 \\
\hline Ring-necked Pheasant & & & 1 & & & & & & & & & & & & & 1 & & & & & & 3 & & \\
\hline Ruffed Grouse & (1) & & & & & & (1) & 3 & 4 & 3 & 3 & & 1 & & 3 & 1 & 1 & (1) & & & 1 & & & 4 \\
\hline Sharp-tailed Grouse & (5) & & 58 & 51 & & 10 & 6 & & & & & 48 & 18 & & & & 1 & & 5 & & 1 & & & 1 \\
\hline Rock Pigeon & & 11 & 317 & 172 & 16 & 96 & 8 & & 75 & 22 & & 610 & 230 & 15 & & & 91 & & & 59 & 10 & 7 & & 50 \\
\hline Eurasian Collared-Dove & & 2 & 19 & 15 & & 2 & 14 & & & & & & 8 & & & & 2 & & 22 & 1 & & 38 & & \\
\hline Bald Eagle & & (1) & & & & 4 & (1) & & & 1 & & 2 & 1 & & & & & & 1 & & 5 & & & 1 \\
\hline Northern Goshawk & & & & & & & & & & & & & & & & & & & 1 & & & 2 & & \\
\hline Golden Eagle & & (1) & & & & & & & & & & & & & & & & & & & & 2 & & \\
\hline Great Horned Owl & (1) & 2 & 1 & 2 & & & & & & 2 & & 4 & 1 & & & & (1) & & 1 & 2 & & 3 & & 1 \\
\hline Snowy Owl & & & & & 1 & & & & & & & 2 & & & & & 4 & (2) & & 1 & & 1 & & \\
\hline Long-eared Owl & & & & & & & & & & & & & & & & & & & & & & & & \\
\hline Downy Woodpecker & 1 & (1) & 6 & 9 & 3 & 5 & 2 & 4 & 2 & 9 & 3 & 17 & 21 & 2 & 1 & 1 & 4 & 3 & 4 & 1 & 1 & 6 & 1 & 4 \\
\hline Hairy Woodpecker & 3 & & 5 & 6 & 2 & 3 & 3 & 6 & 1 & 7 & 4 & 15 & 8 & 3 & 3 & 1 & 3 & 2 & 1 & 2 & 1 & 2 & & 3 \\
\hline $\begin{array}{l}\text { Black-backed } \\
\text { Woodpecker }\end{array}$ & & & & & & & & 1 & & & & & & & & & & & & & & & & \\
\hline Northern Flicker & & (1) & 1 & 1 & & & & & & & & 1 & (1) & 1 & & & & & & & & 7 & & \\
\hline Pileated Woodpecker & (1) & & & & & & & 2 & 1 & & & & & & (1) & & (1) & 1 & & & 2 & & & 1 \\
\hline Merlin & & & 1 & & & & & & & & & 1 & & & & & & & & & & & & \\
\hline Prairie Falcon & & & & & & & & & & & & & & & & & & & & & & & & \\
\hline Northern Shrike & & & & & & & & & & & & 2 & 1 & & & & & & 1 & & & 1 & & \\
\hline Canada Jay & & & & & & & & 2 & & & & & & & & & & 3 & & & 7 & & & 5 \\
\hline Blue Jay & 11 & 1 & 15 & 5 & 3 & 8 & & 19 & 3 & 5 & & 17 & 36 & 4 & 5 & 2 & & 7 & 1 & & 11 & 8 & 2 & \\
\hline Black-billed Magpie & 5 & & 190 & 141 & 17 & 68 & 27 & 10 & 26 & 14 & & 255 & 201 & 27 & 4 & 12 & 46 & & 64 & 29 & 16 & 74 & 41 & \\
\hline American Crow & & & & & & 1 & & & & & & & 1 & & & & & & & & & & & \\
\hline Common Raven & 4 & 5 & 109 & 35 & 13 & 95 & 21 & 115 & 76 & 30 & 3 & 243 & 85 & 12 & 4 & 4 & 14 & & 8 & 15 & 31 & 9 & 40 & 1 \\
\hline Horned Lark & & 40 & 2 & & & & (13) & & & & & 3 & & & & & (2) & & & & & 100 & & \\
\hline Black-capped Chickadee & 30 & & 77 & 49 & 21 & 38 & 18 & 125 & 16 & 43 & 32 & 196 & 204 & 48 & 7 & 43 & 13 & 20 & 59 & 28 & 8 & 26 & 12 & 10 \\
\hline Boreal Chickadee & 1 & & & & & & & 26 & 1 & & & & & & & & & & & & 4 & & & \\
\hline Red-breasted Nuthatch & 1 & 2 & 14 & 23 & & 4 & 3 & 35 & & 8 & 3 & 17 & 7 & 1 & 2 & 5 & & 1 & 8 & 2 & 8 & 27 & & \\
\hline White-breasted Nuthatch & 3 & 1 & & 3 & 3 & & 2 & 5 & 1 & & & 7 & 31 & & 2 & 1 & 1 & 2 & & & & 1 & & 2 \\
\hline Brown Creeper & & & 1 & & & 3 & & & & & & & & & & & & & 2 & 2 & & & & 3 \\
\hline Golden-crowned Kinglet & & & & 2 & & 1 & & 1 & & & & & & & & & & & 2 & 2 & & & & \\
\hline American Robin & & & 4 & & & & 2 & & & & & 7 & 6 & & & 2 & & & & 1 & & & & 4 \\
\hline European Starling & & 3 & 7 & & 9 & & 16 & & & & & 160 & 21 & 12 & & 5 & & (30) & & & & 11 & & 20 \\
\hline Bohemian Waxwing & & & & 22 & 86 & 100 & & & & & & & & & & & 7 & & & & & & & \\
\hline Cedar Waxwing & (6) & & & 1 & & & & & & & & & 10 & & & & & & & & & & & \\
\hline House Sparrow & & 119 & 728 & 604 & 43 & 333 & 90 & & 13 & 144 & 5 & 1523 & 735 & 22 & & 24 & 153 & & 290 & 203 & 4 & 148 & 17 & 6 \\
\hline Evening Grosbeak & 16 & & & & & & & 10 & 12 & & & & & & 14 & & & 18 & & & 13 & & & 35 \\
\hline Pine Grosbeak & 6 & & & & & & & 17 & 13 & & & & & & 8 & & & 15 & & & 1 & & & 1 \\
\hline House Finch & & 2 & 1 & 23 & & & (1) & & & & & 91 & 3 & & & & & & 2 & 2 & & & & \\
\hline Purple Finch & & (1) & & & & & & & & & & & & & & & & & & & & & & \\
\hline Common Redpoll & 59 & & 3 & 12 & 6 & 22 & 70 & 1 & 16 & 27 & 16 & 121 & 56 & & 8 & & 10 & 2 & 2 & & 21 & & & 4 \\
\hline Hoary Redpoll & 6 & & & 1 & & & & & & & & & & & 7 & & (2) & & & & 1 & & & \\
\hline White-winged Crossbill & & & & & & & & 39 & & & & & & & & & & & 7 & 7 & & 12 & & \\
\hline Pine Siskin & & & 28 & 12 & & & (1) & & & & & 15 & & & & & & & 3 & & & 20 & & \\
\hline American Goldfinch & & & & & & & & & & & & & 5 & & & & & & & & & 6 & & \\
\hline Snow Bunting & (20) & & & 27 & & 314 & 110 & & 5 & 16 & & 571 & & & & & 389 & (30) & 378 & & & 123 & 155 & 30 \\
\hline \multicolumn{25}{|l|}{ White-throated Sparrow } \\
\hline Dark-eyed Junco & & 1 & 4 & 3 & & 3 & & & 1 & 1 & & 3 & 10 & & & & & & & & & 7 & & \\
\hline Red-winged Blackbird & & & & & & & & & & & & (1) & & & & & & & & & & & & \\
\hline \multicolumn{25}{|l|}{ Common Grackle } \\
\hline TOTAL BIRDS COUNT DAY & 146 & 197 & 1633 & 1219 & 223 & 1144 & 398 & 426 & 272 & 332 & 69 & 4756 & 1712 & 155 & 68 & 102 & 746 & 74 & 922 & 346 & 476 & 653 & 268 & 200 \\
\hline TOTAL BIRDS ONLY IN CP & 34 & 5 & 0 & 0 & 0 & 0 & 18 & 0 & 0 & 0 & 0 & 1 & 1 & 0 & 1 & 0 & 6 & 63 & 0 & 8 & 0 & 0 & 0 & 0 \\
\hline TOTAL SPECIES COUNT DAY & 13 & 14 & 25 & 23 & 13 & 23 & 16 & 20 & 18 & 15 & 8 & 31 & 25 & 16 & 13 & 13 & 16 & 11 & 23 & 13 & 23 & 28 & 7 & 22 \\
\hline TOTAL SPECIES ONLY IN CP & 6 & 5 & 0 & 0 & 0 & 0 & 6 & 0 & 0 & 0 & 0 & 1 & 1 & 0 & 1 & 0 & 4 & 4 & 0 & 1 & 0 & 0 & 0 & 0 \\
\hline
\end{tabular}



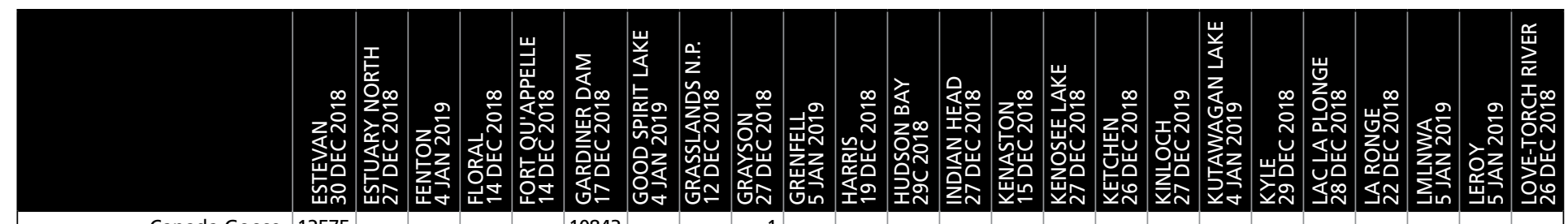
12575

Common Goldeneye Mallard Gray Partridge Ring-necked Pheasant Ruffed Grouse

Sharp-tailed Grouse Rock Pigeon Eurasian Collared-Dove Bald Eagle Northern Goshawk Golden Eagle Great Horned Owl Snowy Owl Long-eared Owl

Downy Woodpecker Hairy Woodpecker Black-backed Woodpecker

Northern Flicker Pileated Woodpecker Merlin Prairie Falcon Northern Shrike Canada Jay Blue Jay

Black-billed Magpie American Crow Common Raven Horned Lark Black-capped Chickadee Boreal Chickadee Red-breasted Nuthatch White-breasted Nuthatch Brown Creeper Golden-crowned Kinglet American Robin

European Starling

Bohemian Waxwing Cedar Waxwing House Sparrow Evening Grosbeak Pine Grosbeak House Finch Purple Finch

Common Redpoll Hoary Redpoll White-winged Crossbill Pine Siskin

American Goldfinch Snow Bunting White-throated Sparrow Dark-eyed Junco Red-winged Blackbird

Common Grackle

TOTAL BIRDS COUNT DAY TOTAL BIRDS ONLY IN CP TOTAL SPECIES COUNT DAY TOTAL SPECIES ONLY IN CP

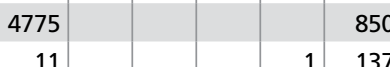

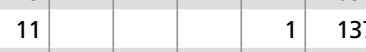

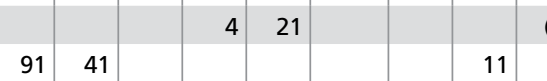

(6)

31

32

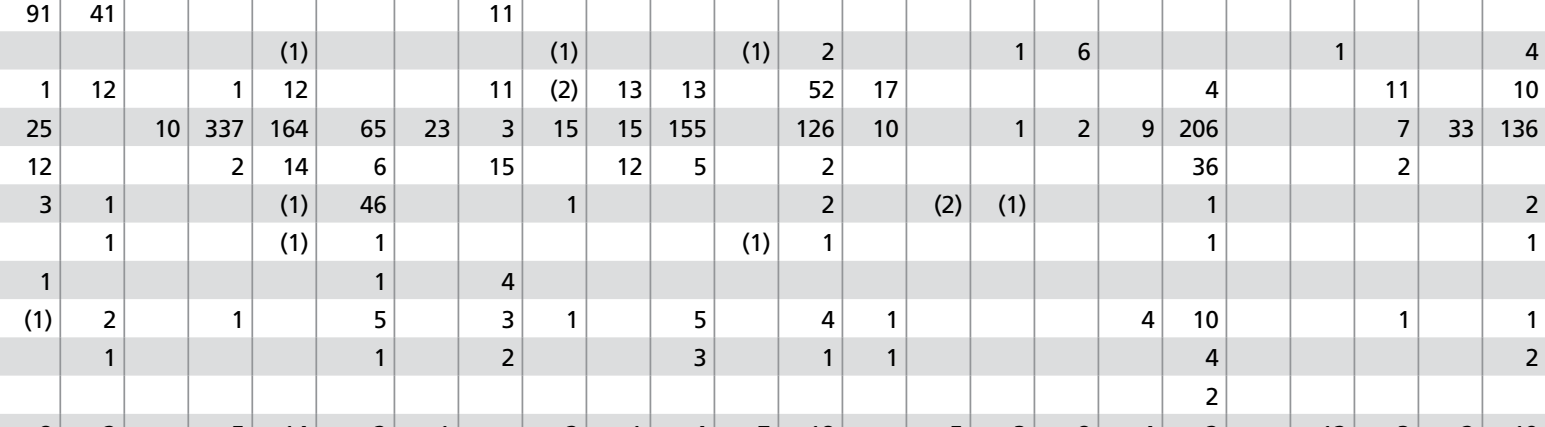

\begin{tabular}{|l|l|l|l|l|l|l|l|l|l|l|l|l|l|l|l|l|l|l|l|l|l|l|l|l|}
\hline 8 & 2 & 5 & 14 & 2 & 1 & 2 & 1 & 4 & 7 & 18 & 5 & 3 & 8 & 4 & 2 & 12 & 3 & 2 & 19 \\
\hline
\end{tabular} \begin{tabular}{|l|l|l|l|l|l|l|l|l|l|l|l|l|l|l|l|}
6 & 4 & 2 & 9 & 4 & 2 & 2 & 1 & 1 & 11 & 17 & (1) & 8 & 3 & 1 \\
\hline
\end{tabular}

(1)

(1)

(1)

(1)

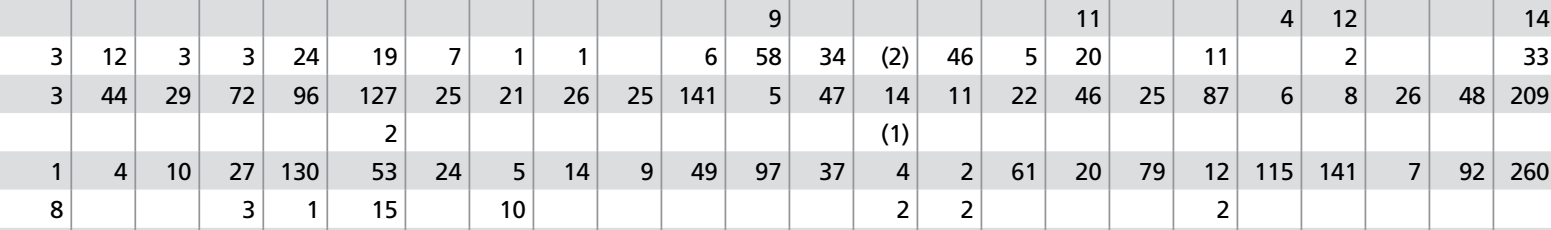
\begin{tabular}{|l|l|l|l|l|l|l|l|l|l|l|l|l|l|l|l|l|l|l|l|l|l|l|l|l|l|l|}
\hline 18 & 14 & 11 & 31 & 166 & 43 & 8 & 20 & 6 & 23 & 69 & 146 & 16 & 16 & 44 & 21 & 7 & 5 & 37 & 25 & 19 & 79 \\
\hline
\end{tabular}

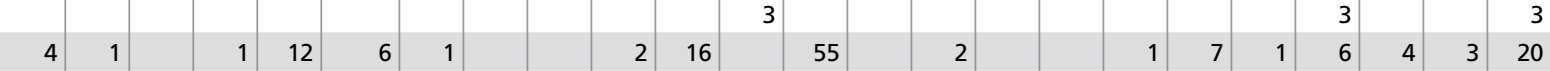

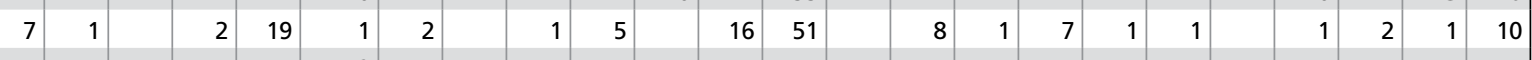

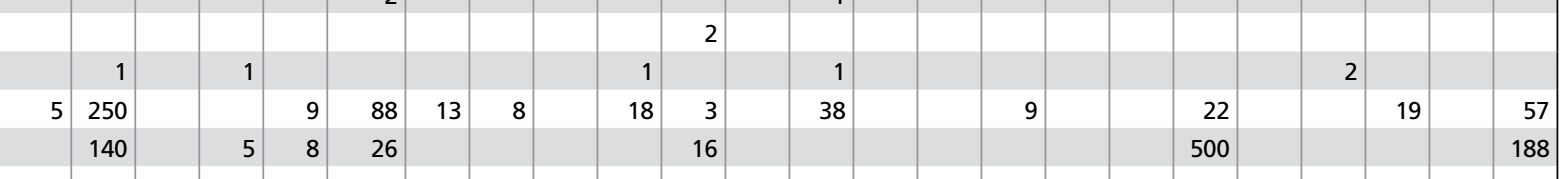

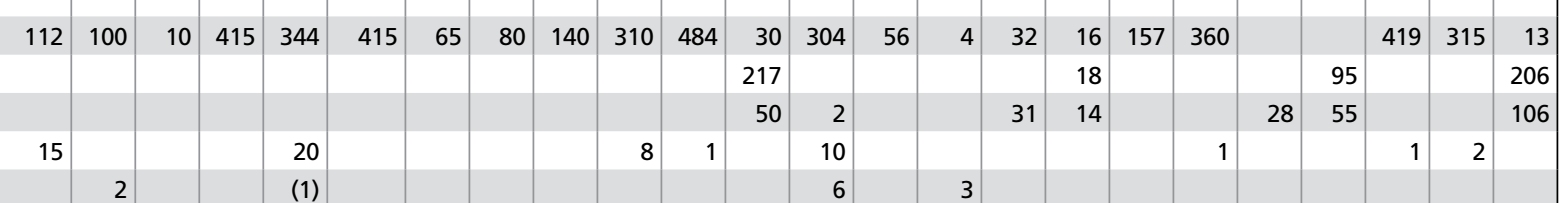

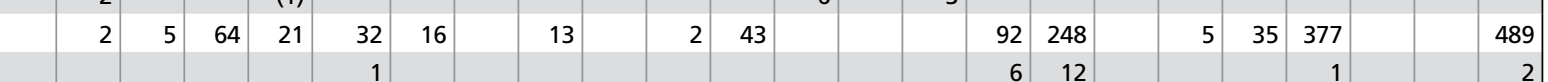

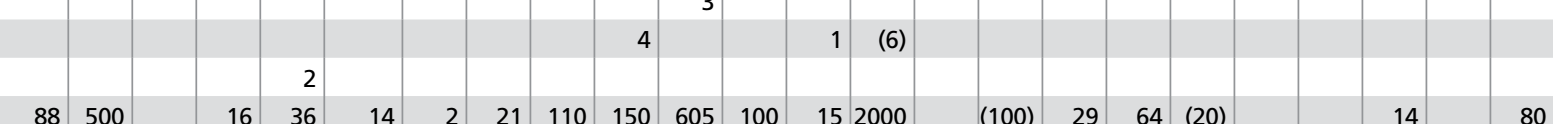
\begin{tabular}{|l|l|r|r|r|r|r|r|r|r|r|r|r|r|r|r|r|r|r|r|r|}
\hline 88 & 500 & 16 & 36 & 14 & 2 & 21 & 110 & 150 & 605 & 100 & 15 & 2000 & $(100)$ & 29 & 64 & $(20)$ & 14 \\
\hline
\end{tabular} \begin{tabular}{|l|l|l|l|l|l|l|l|l|l|l|l|}
6 & 4 & $(1)$ & 5 & 1 & 5 & 6 & 10 & 2 & 12 \\
\hline
\end{tabular}

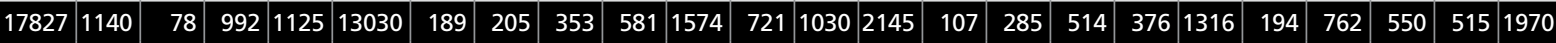

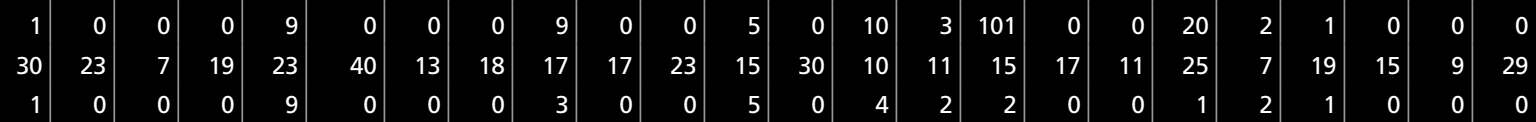


TABLE 3. Species found on 6 or more counts; ()$=$ seen during count period (CP); CP totals include CP birds listed in Table 4.

\begin{tabular}{|c|c|c|c|c|c|c|c|c|c|c|c|c|c|c|c|c|c|c|c|c|c|c|c|c|}
\hline SPECIES & 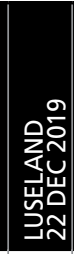 & 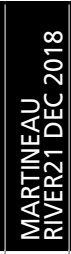 & 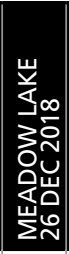 & 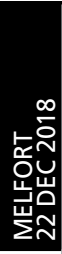 & 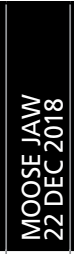 & 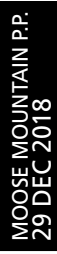 & 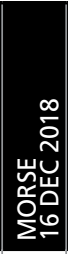 & 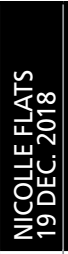 & 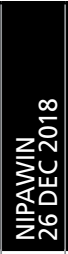 & 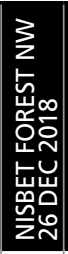 & 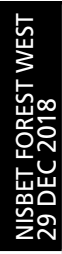 & 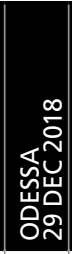 & 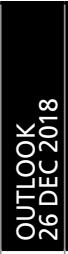 & 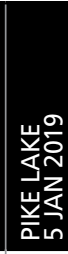 & 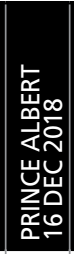 & 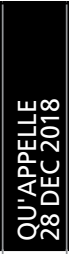 & 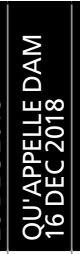 & 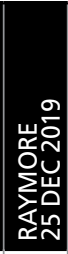 & 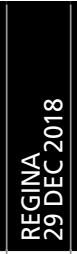 & 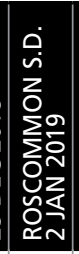 & 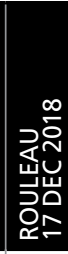 & 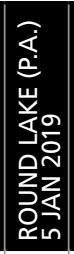 & 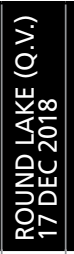 & 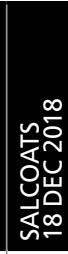 \\
\hline Canada Goose & & & & & & & (70) & & & & & & $(67)$ & & & & 1119 & & 65 & & & & 2 & \\
\hline Mallard & & 9 & & & & & & & & & & & & & & & 7 & & 135 & & & & 10 & \\
\hline Common Goldeneye & & 3 & & & & & & & & & & & 2 & & & & 322 & & (1) & & & & & \\
\hline Gray Partridge & 75 & & & & & & 217 & 57 & & & & 15 & & & & 11 & 12 & 10 & 120 & & (12) & & 7 & 10 \\
\hline Ring-necked Pheasant & & & & & 1 & & 3 & & & & & & & & & & & & & & & & & \\
\hline Ruffed Grouse & & 3 & & & & 2 & & & 1 & (1) & 5 & 2 & & 1 & 1 & 4 & & & & 2 & & & & 3 \\
\hline Sharp-tailed Grouse & 9 & & & & & & 35 & 16 & & & & 20 & & 52 & & 64 & 5 & & 2 & & & & & \\
\hline Rock Pigeon & 75 & & 5 & 19 & 512 & 2 & 167 & 48 & 170 & & 55 & 10 & 18 & 136 & 344 & 69 & 21 & 56 & 1249 & (71) & 148 & & 13 & 20 \\
\hline Eurasian Collared-Dove & 11 & & & 3 & 112 & & 21 & & & & & & 17 & & & 1 & 3 & & 4 & & & & & 6 \\
\hline Bald Eagle & & & & & & & (1) & & & & & 4 & & 3 & & & 12 & & & & & & 2 & 6 \\
\hline Northern Goshawk & & & & & & & & & & & & & 1 & & & & & & & & & & & \\
\hline Golden Eagle & & & & & & & & & & & & & 1 & & & & 1 & & & & & & & 1 \\
\hline Great Horned Owl & 6 & & & & 4 & 1 & 17 & 3 & & & (1) & 2 & & 4 & & (1) & 6 & (1) & 7 & (1) & 3 & & & \\
\hline Snowy Owl & 4 & & & (2) & 9 & & 7 & & & & 1 & 2 & & & & & & & 16 & (1) & 12 & & & \\
\hline Long-eared Owl & & & & & & & & & & & & & & & & & 1 & & & & & & & \\
\hline Downy Woodpecker & (1) & & & 1 & 7 & 3 & 4 & 3 & 6 & 2 & 2 & 4 & & 28 & 13 & 17 & 7 & 1 & 37 & 8 & & & 6 & 14 \\
\hline Hairy Woodpecker & 1 & 1 & & 2 & 7 & 1 & 1 & 1 & 5 & 2 & 3 & 4 & & 19 & 6 & 12 & 6 & 1 & 4 & 17 & & 1 & 5 & 14 \\
\hline $\begin{array}{l}\text { Black-backed } \\
\text { Woodpecker }\end{array}$ & & & 1 & & & & & & & & & & & & (2) & & & & 1 & & & & & \\
\hline Northern Flicker & (1) & & & & & & & 1 & & & & & & & 3 & & 2 & & 10 & & & & & \\
\hline Pileated Woodpecker & & & & & & 1 & & & & & & 2 & & 5 & & & 1 & & & & & & & \\
\hline Merlin & & & & & 1 & & & & & & & & & & & & & & 2 & & & & & \\
\hline Prairie Falcon & & & & & & & & & & & & & & & & & 1 & & 1 & & & & & \\
\hline Northern Shrike & & & & & & & 3 & & & & & & & (1) & & 1 & & & 2 & & & & 1 & \\
\hline Canada Jay & & 10 & & & & & & & (1) & & & & & & 9 & & & & & & & & & \\
\hline Blue Jay & 5 & 20 & & 1 & 3 & 9 & 2 & 3 & 33 & 3 & 14 & & 5 & 28 & 42 & & 10 & & 12 & 12 & & 2 & 12 & 17 \\
\hline Black-billed Magpie & 105 & 2 & 15 & 41 & 66 & 10 & 41 & 28 & 15 & 2 & 13 & 20 & 97 & 144 & 133 & 96 & 73 & 30 & 90 & 47 & 8 & & 24 & 6 \\
\hline American Crow & & & & & 1 & & & & & & & & & & & & & & & & & & & \\
\hline Common Raven & 11 & 29 & 30 & 82 & 21 & 6 & 8 & 32 & 88 & 5 & 11 & 10 & 4 & 69 & 202 & 38 & 22 & 41 & 215 & 36 & 17 & 2 & 16 & 52 \\
\hline Horned Lark & 58 & & & & 3 & & 83 & 1 & & & & & & & & 2 & & 17 & & & & & 2 & \\
\hline Black-capped Chickadee & 3 & 28 & 6 & 34 & 37 & 40 & & 12 & 61 & 36 & 31 & 20 & 4 & 409 & 230 & 67 & 54 & 21 & 251 & 54 & & 12 & 11 & 94 \\
\hline Boreal Chickadee & & 17 & & & & & & & & & & & & & 4 & & & & & & & & & \\
\hline Red-breasted Nuthatch & 7 & 10 & & 10 & 14 & 2 & 15 & & 18 & 1 & & 4 & 1 & 12 & 14 & 2 & 5 & 1 & 178 & 2 & & 1 & 1 & 2 \\
\hline White-breasted Nuthatch & 1 & 1 & & 2 & 18 & 2 & 1 & & 8 & 2 & & & & 25 & 10 & 3 & 5 & 1 & 34 & 11 & & & 2 & 11 \\
\hline Brown Creeper & & & & (1) & & & & & & & & & & & & & & & 1 & & & & & \\
\hline Golden-crowned Kinglet & & & & & & & & & & & & & & & & & & & 2 & & & & & \\
\hline American Robin & & & & & & & & & & & & 2 & & & 2 & & 6 & & 6 & & & & & \\
\hline European Starling & & & & (30) & 14 & & 22 & & 40 & & & & & 125 & & 1 & 88 & & 43 & & & & & \\
\hline Bohemian Waxwing & & & 100 & 40 & & & & & 147 & & (20) & & & 10 & 1318 & & 206 & & & 21 & & & & \\
\hline Cedar Waxwing & & & & & & & & & & & & & & & & & & & 22 & & & & & \\
\hline House Sparrow & 107 & & & 180 & 463 & 19 & 1323 & 30 & 103 & 24 & 30 & 40 & 84 & 330 & 502 & 263 & 376 & 80 & 3750 & 60 & 47 & & 228 & 220 \\
\hline Evening Grosbeak & & & & & & & & & 45 & & 32 & & & & 26 & & & & & & & 8 & & \\
\hline Pine Grosbeak & & & & 12 & & & & & 48 & 1 & 12 & 2 & & & 53 & & & & & 26 & & 6 & & \\
\hline House Finch & & & & 10 & 46 & & & & & & & & & 1 & & 3 & 14 & & 242 & & & & & \\
\hline Purple Finch & & & & & & & & 3 & & & & & & & & (1) & & & 2 & & & & & 10 \\
\hline Common Redpoll & 120 & 80 & & 23 & & & 13 & & 45 & & 192 & & & 97 & 79 & 176 & 34 & & 2 & 6 & & 52 & & 16 \\
\hline Hoary Redpoll & & & & 2 & & & 1 & & & & & & & & & & & & & & & & & \\
\hline White-winged Crossbill & & 32 & & & & & & & & & & & & 10 & 4 & & & & & & & & & \\
\hline Pine Siskin & & & & 10 & 12 & & & & 4 & & & & & & 17 & & & & 39 & & & & & 16 \\
\hline American Goldfinch & & & & & & & & & & & (6) & & & & 15 & & & & 2 & & & & & \\
\hline Snow Bunting & 574 & & 50 & 55 & 62 & & 71 & & & & 40 & & & 157 & 30 & 50 & 2 & & & (155) & 145 & & 30 & \\
\hline White-throated Sparrow & & & & 1 & & & & & & & & & 1 & & & 2 & 1 & & 5 & & & & & \\
\hline Dark-eyed Junco & & & & & 9 & & 6 & & 2 & & & 6 & & 2 & 1 & 1 & & & 70 & 10 & & & 3 & \\
\hline Red-winged Blackbird & & & & & & & & & & & & & & & & & 1 & & & & & & & \\
\hline Common Grackle & & & & & 3 & & & & & & & & & & & & & & 1 & & & & & \\
\hline TOTAL BIRDS COUNT DAY & 1172 & 245 & 207 & 529 & 1426 & 98 & 2061 & 239 & 839 & 78 & 441 & 175 & 235 & 1668 & 3061 & 890 & 2425 & 259 & 6636 & 312 & 380 & 84 & 375 & 519 \\
\hline TOTAL BIRDS ONLY IN CP & 2 & 0 & 0 & 33 & 1 & 0 & 72 & 0 & 1 & 1 & 27 & 0 & 67 & 1 & 2 & 2 & 0 & 1 & 1 & 228 & 12 & 0 & 0 & 0 \\
\hline TOTAL SPECIES COUNT DAY & 17 & 14 & 7 & 20 & 24 & 13 & 22 & 15 & 18 & 10 & 14 & 20 & 12 & 22 & 27 & 23 & 32 & 11 & 41 & 14 & 7 & 8 & 18 & 19 \\
\hline TOTAL SPECIES ONLY IN CP & 2 & 0 & 0 & 3 & 1 & 0 & 3 & 0 & 1 & 1 & 3 & 0 & 1 & 1 & 1 & 2 & 0 & 1 & 1 & 4 & 1 & 0 & 0 & 0 \\
\hline
\end{tabular}



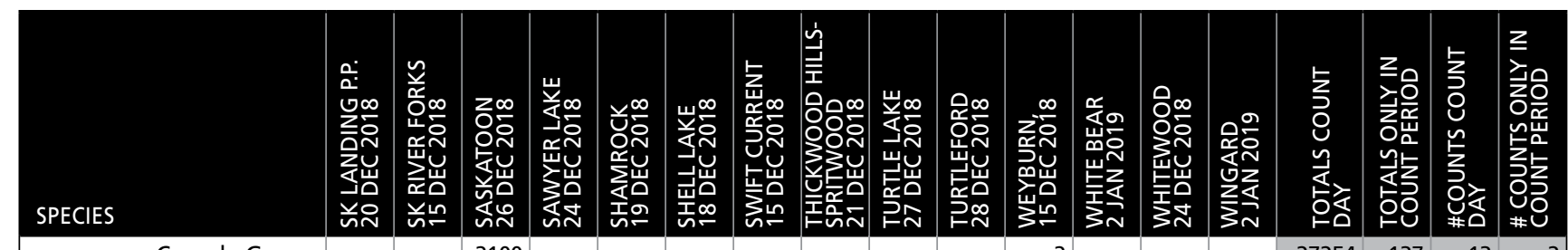

Gray Partridge

Ring-necked Pheasant

Ruffed Grouse

Sharp-tailed Grouse

Rock Pigeon

Eurasian Collared-Dove Bald Eagle

Northern Goshawk

Golden Eagle

Great Horned Owl Snowy Owl

Long-eared Owl

Downy Woodpecker

Hairy Woodpecker

Black-backed

Woodpecker

Northern Flicker

Pileated Woodpecker Merlin

Prairie Falcon

Northern Shrike

Canada Jay Blue Jay

Black-billed Magpie

American Crow

Common Raven

Horned Lark

Black-capped Chickadee

Boreal Chickadee

Red-breasted Nuthatch

White-breasted Nuthatch

Brown Creeper

Golden-crowned Kinglet American Robin

European Starling

Bohemian Waxwing

Cedar Waxwing

House Sparrow

Evening Grosbeak

Pine Grosbeak

House Finch

Purple Finch

Common Redpoll

Hoary Redpoll

White-winged Crossbill Pine Siskin

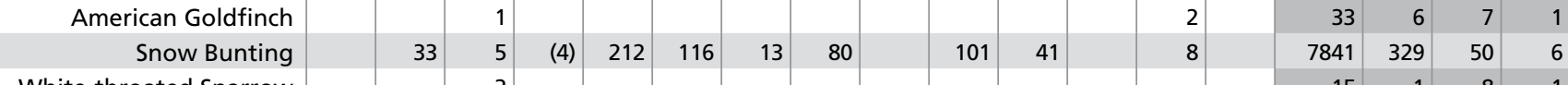

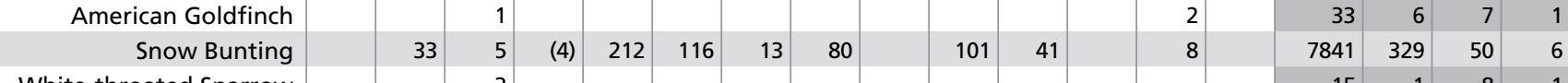
85

178

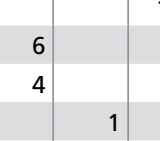

(10)

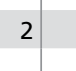

2

(1)

\begin{tabular}{|l|l|l|l|}
27254 & 137 & 13 & 2 \\
\hline
\end{tabular}

\begin{tabular}{|l|l|l|l|}
\hline 5915 & 1 & 12 & 1 \\
\hline
\end{tabular}

\begin{tabular}{|l|l|l|l|}
\hline 1213 & 1 & 11 & 1 \\
\hline
\end{tabular}

\begin{tabular}{|l|l|l|l|}
1202 & 36 & 34 & 4 \\
\hline
\end{tabular}

\begin{tabular}{|r|r|r|r|}
199 & 0 & 12 & 0 \\
\hline
\end{tabular}

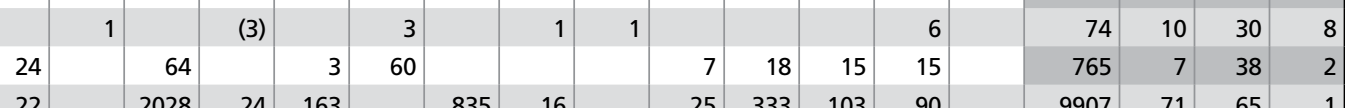

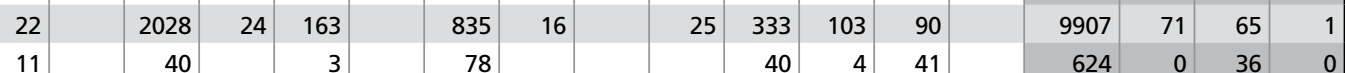

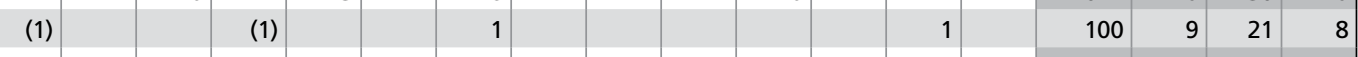

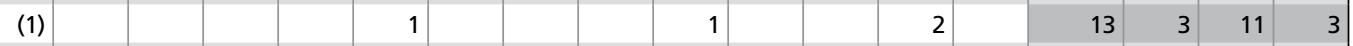

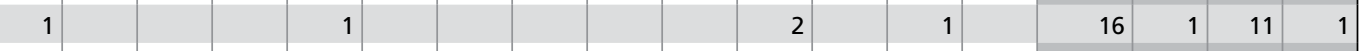

\begin{tabular}{|l|r|r|r|r|r|r|r|r|r|r|r|r|r|r|}
\hline 5 & 2 & 11 & 2 & 3 & & 2 & 3 & 9 & 1 & 148 & 7 & 41 & 7 \\
\hline
\end{tabular}

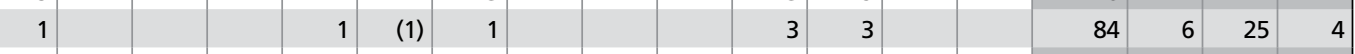

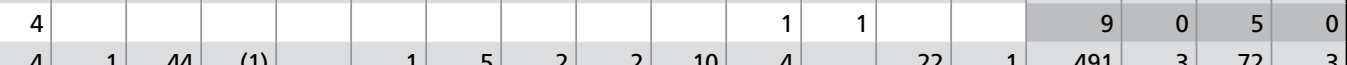

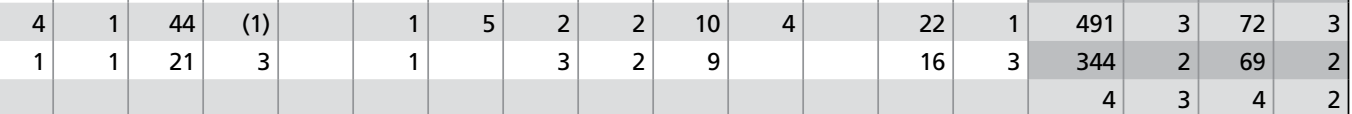

$1 \quad \mid 16$

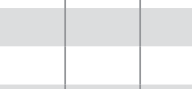

6

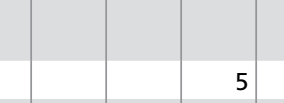

(2) 2

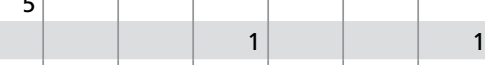

\begin{tabular}{l|l|l|l|}
50 & 4 & 13 & 4 \\
\hline & 5 & 15 & 5
\end{tabular}

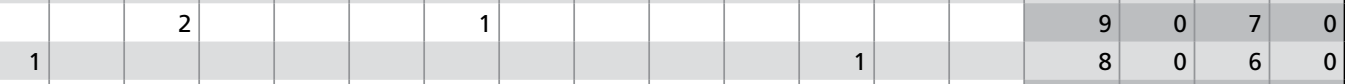

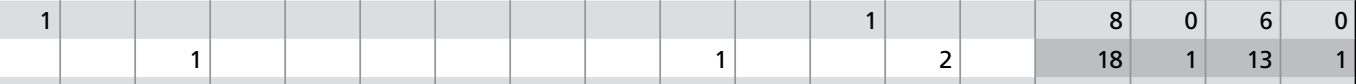

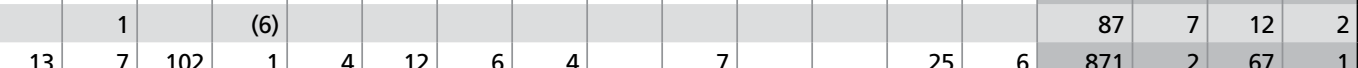

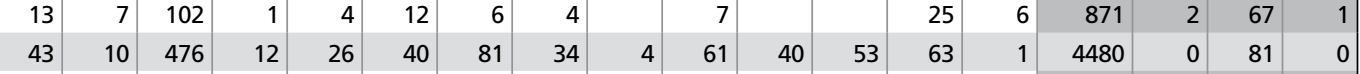

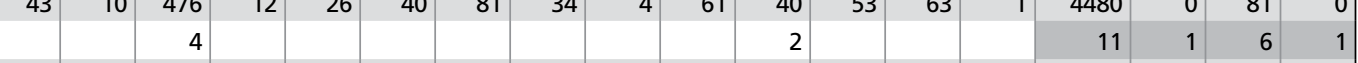
\begin{tabular}{|l|l|l|l|l|l|l|l|l|l|l|l|l|l|l|l|l|}
\hline 5 & 22 & 128 & 18 & 5 & 26 & 1 & 42 & 22 & 74 & 29 & 62 & 3706 & 0 & 83 & 0 \\
\hline
\end{tabular}

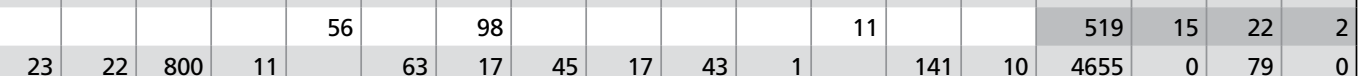
\begin{tabular}{|l|l|l|l|l|l|l|l|l|l|l|l|r|r|r|r|r|r|}
\hline 23 & 22 & 800 & 11 & 63 & 17 & 45 & 17 & 43 & 1 & & 141 & 10 & 4655 & 0 & 79 & 0 \\
\hline
\end{tabular} \begin{tabular}{|l|l|l|l|l|l|l|l|l|l|l|l|l|r|r|r|}
\hline 1 & 143 & 5 & 2 & 32 & 1 & 2 & 7 & 14 & 3 & 823 & 0 & 66 & 0 \\
\hline
\end{tabular} \begin{tabular}{|r|r|r|r|r|r|r|r|r|r|r|r|r|r|r|r|r|r|}
\hline 3 & 2 & 24 & $(1)$ & & 4 & 2 & 4 & 2 & 7 & 17 & 404 & 1 & 60 & 1 \\
\hline
\end{tabular}

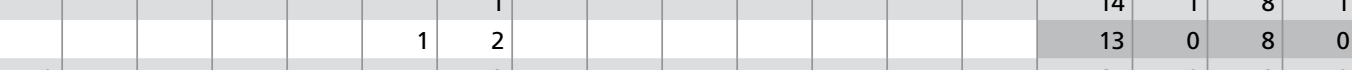

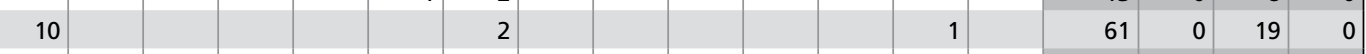
\begin{tabular}{|l|r|r|r|r|r|r|r|r|r|r|r|r|}
\hline 69 & 14 & 6 & 20 & & 44 & 24 & 1313 & 60 & 36 & 2 \\
\hline
\end{tabular}

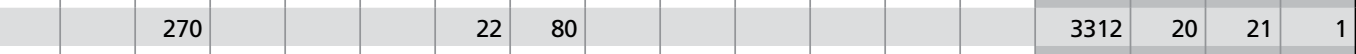

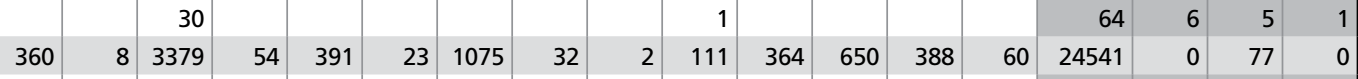

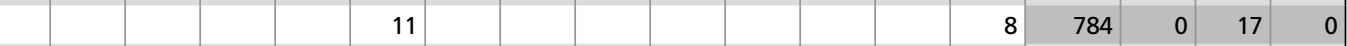
\begin{tabular}{|l|l|l|l|l|l|l|l|l|l|l|l|r|r|r|r|r|}
\hline 8 & 8 & & 13 & & 13 & & $(6)$ & & & 6 & 555 & 6 & 27 & 1 \\
\hline & 353 & & & & 79 & & & & & & 1 & & 931 & 1 & 24 & 1 \\
\hline
\end{tabular}

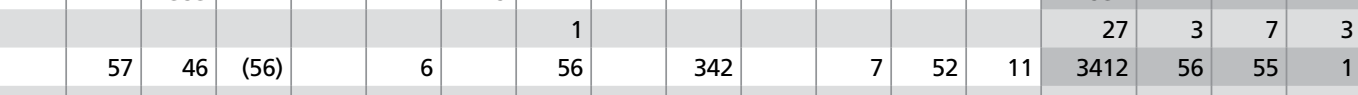

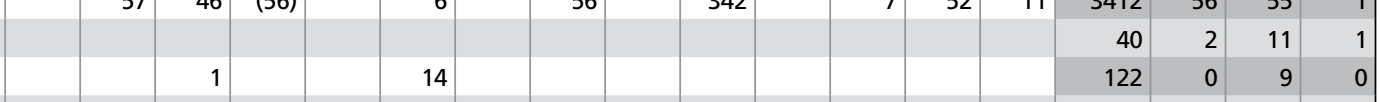

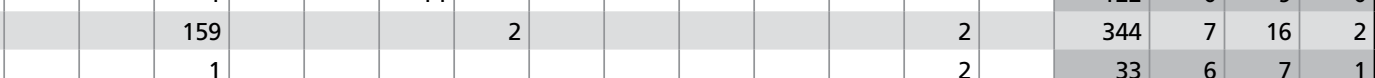
White-throated Sparrow Dark-eyed Junco

Red-winged Blackbird

Common Grackle

TOTAL BIRDS COUNT DAY

TOTAL BIRDS ONLY IN CP

TOTAL SPECIES COUNT DAY

TOTAL SPECIES ONLY IN CP

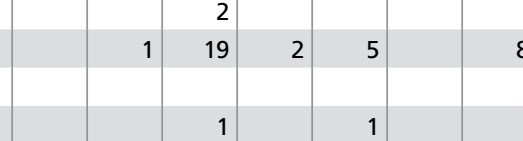

\begin{tabular}{|l|r|r|}
\hline 8 & 14 & (3) \\
\hline
\end{tabular}

(3)

\begin{tabular}{|l|l|r|r|}
15 & 1 & 8 & 1 \\
\hline
\end{tabular}

\begin{tabular}{|r|r|r|r|r|r|r|r|r|r|r|r|r|r|r|r|r|r|}
\hline 614 & 174 & 10557 & 133 & 977 & 428 & 2462 & 414 & 50 & 800 & 1111 & 867 & 998 & 109 & 108164 & 846 & & \\
2 & 0 & 2 & 82 & 0 & 1 & 0 & 0 & 0 & 6 & 0 & 0 & 4 & 0 & 846 & & \\
24 & 14 & 39 & 9 & 18 & 20 & 28 & 16 & 7 & 18 & 22 & 12 & 26 & 10 & 88 & & \\
2 & 0 & 1 & 8 & 0 & 1 & 0 & 0 & 0 & 1 & 0 & 0 & 2 & 0 & & 3 & \\
\hline
\end{tabular}


TABLE 4. Species found in fewer than 6 counts.

\begin{tabular}{|c|c|}
\hline SPECIES & LOCALITY AND NUMBER (*=SEEN DURING COUNT PERIOD) \\
\hline Cackling Goose & Estevan (41), Gardiner Dam (17) \\
\hline Wood Duck & Regina (1) \\
\hline Gadwall & Estevan (1) \\
\hline Northern Pintail & Grayson (1), Crooked Lake \\
\hline Green-winged Teal & Gardiner Dam (1) \\
\hline Redhead & Gardiner Dam (15) \\
\hline Ring-necked Duck & Gardiner Dam (10), Saskatoon (2) \\
\hline Greater Scaup & Gardiner Dam (20) \\
\hline Lesser Scaup & Estevan (1), Gardiner Dam (77), Saskatoon (5) \\
\hline Bufflehead & Gardiner Dam (4) \\
\hline Common Merganser & $\begin{array}{l}\text { Clarks Crossing (3), E.B.Campbell Dam (1), } \\
\text { Gardiner Dam (87), La Ronge (1*) }\end{array}$ \\
\hline Greater Sage-Grouse & Grasslands National Park (4) \\
\hline Spruce Grouse & Lac La Plonge $(1 *)$ \\
\hline Willow Ptarmigan & La Ronge (1) \\
\hline Mourning Dove & Harris (1), Moose Jaw (1), Prince Albert (1), Saltcoats (1) \\
\hline American Coot & Estevan (2) \\
\hline Double-crested Cormorant & Gardiner Dam (1) \\
\hline American White Pelican & Gardiner Dam (2) \\
\hline Great Blue Heron & Regina (1) \\
\hline Sharp-shinned Hawk & $\begin{array}{l}\text { Ft. Qu'Appelle (1*), Melfort (1), Prince Albert (1), } \\
\text { Saskatoon }(1 *)\end{array}$ \\
\hline Cooper's Hawk & Indian Head (1) \\
\hline Red-tailed Hawk & Eastend (1), Ft. Qu'Appelle (1*) \\
\hline Rough-legged Hawk & $\begin{array}{l}\text { Balgonie (1), Broadview (1*), Eastend (1), } \\
\text { Grasslands National Park (4) }\end{array}$ \\
\hline Northern Hawk Owl & Candle Lake (2) \\
\hline Short-eared Owl & Regina (6) \\
\hline Northern Saw-whet Owl & Morse $(1 *)$ \\
\hline American Three-toed Woodpecker & $\begin{array}{l}\text { Candle Lake (3), Hudson Bay (1*), Love-Torch River (1), } \\
\text { Saskatoon (1) }\end{array}$ \\
\hline Gyrfalcon & Borden-Radisson (1), Crooked Lake (1), Gardiner Dam (1) \\
\hline Peregrine Falcon & Ft. Qu'Appelle (1*) \\
\hline Townsend's Solitaire & Moose Jaw (1*), Qu'Appelle (1), Qu'Appelle Dam (1) \\
\hline Varied Thrush & Borden-Radisson (1), Saskatoon (1) \\
\hline Red Crossbill & E.B.Campbell Dam (8), Saskatoon (7) \\
\hline Spotted Towhee & Saskatoon $(1 *)$ \\
\hline American Tree Sparrow & $\begin{array}{l}\text { Borden-Radisson (4), Eastend (7), Qu'Appelle (6), } \\
\text { Saskatchewan Landing (1) }\end{array}$ \\
\hline White-crowned Sparrow & Prince Albert (1) \\
\hline Rusty Blackbird & Nicolle Flats (1), Odessa (2), Saskatchewan Landing (1) \\
\hline Brewer's Blackbird & Weyburn (1) \\
\hline
\end{tabular}

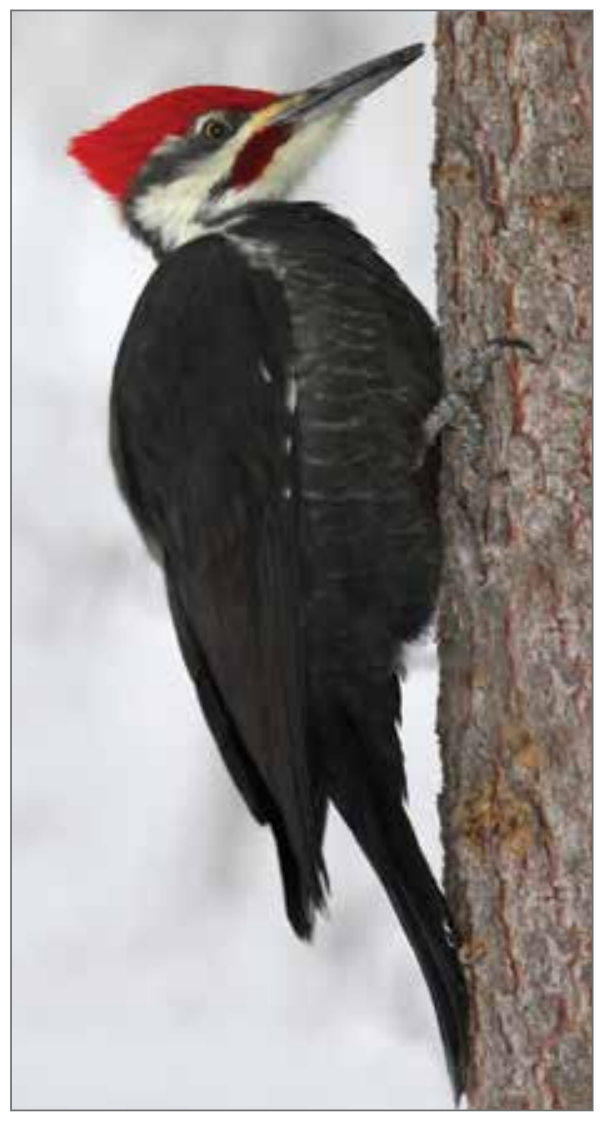

Pileated Woodpecker. Photo Credit Nick Saunders

\section{TABLE 5. Birds not identified to species.}

\begin{tabular}{|l|l|}
\hline CATEGORY & $\begin{array}{l}\text { LOCALITY AND NUMBER } \\
\text { (*=SEEN DURING } \\
\text { COUNT PERIOD) }\end{array}$ \\
\hline Teal sp. & $\begin{array}{l}\text { Regina (1) } \\
\text { Regina (1) }\end{array}$ \\
Small Accipiter & $\begin{array}{l}\text { Avonlea (1) } \\
\text { Clarks Crossing (1) } \\
\text { Hawk sp. }\end{array}$ \\
Saskatoon (1) \\
Woodpecker sp. & Pike Lake (1) \\
\hline
\end{tabular}

\section{TABLE 6. New (in bold and italics) and tying high counts for individual species 2018.}

\begin{tabular}{|l|l|l|l|l|}
\hline LOCATION & 2018 COUNT & SPECIES & $\begin{array}{l}\text { PREVIOUS } \\
\text { HIGH }\end{array}$ & LOCATION, YEAR \\
\hline Gardiner Dam & $\mathbf{1 0}$ & Ring-necked Duck & $\mathbf{7}$ & $\begin{array}{l}\text { Gardiner Dam 2011 } \\
\text { Gardiner Dam 2004 }\end{array}$ \\
\hline Gardiner Dam & $\mathbf{7 7}$ & Lesser Scaup & $\mathbf{2 4}$ & $\begin{array}{l}\text { 6 previous counts north to Grand Centre-Pierceland } \\
\text { and Squaw Rapids }\end{array}$ \\
\hline Gardiner Dam & 1 & Double-crested Cormorant & 1 & 5 previous counts north to Barrier Lake \\
\hline Regina & 1 & Great Blue Heron & 1 & 13 previous counts north to Turtle Lake \\
\hline Saskatoon & 1 & Spotted Towhee & 1 & \\
\hline
\end{tabular}

